EMERITA. Revista de Lingüística y Filología Clásica (EM)

LXXIV 2, julio-diciembre de 2006

pp. 233-258

ISSN 0013-6662

\title{
BEMERKUNGEN ZUM LESBISCHEN DIALEKTALEN WORTSCHATZES
}

\author{
WoJCIECH SOWA \\ Jagellonen Universität Krakau \\ wojciech.sowa@gazeta.pl
}

Lesbisch scheint eine besondere Rolle unter den griechischen Dialekten zu spielen. Einerseits ist es durch eine große Menge epigraphischer Denkmäler bezeugt, andererseits kommt es auch als literarische Varietät in der lyrischen Orginalliteratur von Sappho und Alkaios vor. Diese bis zu einem bestimmten Ausmaß in dem Vernaculardialekt geschriebene Poetik scheint einen großen Einfluß auf die anderen poetischen Werke ausgeübt zu haben, vor allem auf die archaische Dichtung, auch auf die hellenistische, dann auch auf die römische Literatur. Die folgende Studie wird den Problemen des lesbischen dialektalen Wortschatzes gewidmet. Das Hauptziel solcher Untersuchung ist ein Bild des lesbischen Lexikons durch Vergleich der epigraphischen Daten mit den bezeugten Glossen zu geben. Bei der Beschreibung der Probleme werden auch verschiedene Ebenen des Wortschatzes unterscheidet: Formen, die gemeinsam für alle griechischen Dialekte sind, werden den, die dialektspezifisch angesehen werden können, gegenübergestellt. Selbstverständlich scheint die exakte Antwort auf die Frage, ob eine bestimmte Form als ein typisches Dialektalelement betrachtet werden kann oder nicht, in vielen Fällen unmöglich zu sein. Deswegen sind auch die Hinweise auf ein mögliches Auftreten der Form in anderen Dialekten, bzw. die Geschichte des Wortes, auch die Tatsache, ob es ausschließlich in den Inschriften, oder auch in der lesbischen Literatur auftritt, wichtig.

Schlüssel-Wörter: historische Sprachwissenschaft, Altgriechisch, griechische Dialekte, Lesbisch, Wortschatz
Lesbian plays a specific role among the Greek dialects. On the one hand it is attested in a large number of epigraphical documents, on the other hand it occurs also in a literary variant, in the original lyric poetry of Sappho and Alcaeus. This poetry, written to some extent in the vernacular dialect, had much influence upon other Greek literary works, first of all upon archaic poetry, then also upon that of the Hellenistic period, then also upon Roman literature. The following paper will be devoted to the problems of Lesbian dialectal vocabulary. The main aim of such a study is to try to give a picture of the Lesbian lexicon by confronting the epigraphical data with the attested glosses, to try to point out the different levels in the vocabulary: the forms common to all Greek dialects, the specifically dialectal ones, etc. Of course, in many cases, the precise answer to the question of whether the form can or cannot be considered dialectal seems impossible. The most important thing seems to me the possible reference to the other dialects, or the history of a word, whether it occurs exclusively in inscriptions or whether it is also attested in the literature.

Key-words: historical linguistics, Ancient Greek language, Greek dialects, Lesbian, lexicon 
Lesbisch scheint eine besondere Rolle unter den griechischen Dialekten zu spielen. Einerseits ist es durch eine große Menge epigraphischer Denkmäler bezeugt, andererseits kommt es auch als literarische Varietät in der lyrischen Orginalliteratur von Sappho und Alkaios vor. Diese bis zu einem bestimmten Aus$\mathrm{ma} ß$ in dem Vernaculardialekt geschriebene Poetik scheint einen großen Einfluß auf die anderen poetischen Werke ausgeübt zu haben, vor allem auf die archaische Dichtung, dann auch auf die hellenistische Periode - vgl. die drei in literarischem Lesbisch verfaßten Idylle des Theokrit -, dann auch auf die römische Literatur, die das äolische Metrum und äolische Motive übernimmt. Die Epigramme von Balbilla, die im lesbischen Dialekt verfaßt sind, bezeugen, daß dieser noch im 2. Jh. nach Christus populär war. Diese ständige Wechselwirkung zwischen epigraphischer und literarischer Tradition hat auch im Lexikon ihren Niederschlag gefunden (vgl. Hodot 1990, Ss. 66-69). Die Quellen des Lesbischen umfassen zunächst 500 Dialektinschriften, die vom 7. Jh. vor bis zum 3. Jh. n.Chr. bezeugt sind. Sie stammen aus den Gebieten von Lesbos (Mytilene, Methymna, Eressos, Antissa), Nesos, Tenedos und aus der äolischen Region Kleinasiens, z.B. aus Assos, Kyme oder Myrina. Archaische Inschriften sind äußerst selten, sie tragen aber ohnehin wenig zur Lösung von Fragen des Lexikons bei ${ }^{1}$. Die Mehrheit der Dokumente stammt aus dem Zeitraum zwischen dem 3. und 2. Jh. v.Chr. und aus der römischen Periode (464 Dokumente von der Gesamtzahl von 524; vgl. Hodot 1990, S. 18). Es ist mithin zu vermuten, daß man immer mit der Möglichkeit eines Einflusses der кoเví zu rechnen hat, und die Dokumente aus der Kaiserzeit bieten Formen, die als Ergebnis archaisierender Tendenzen zu Hadrians Zeiten angesehen werden können. Die Dialektismen scheinen eher Zeugnisse einer Stilisierung nach dem literarischen Dialekt zu sein und sollten nicht als Zeugnisse der lingua uernacula jener Zeit betrachtet werden (Hodot 1990, S. 19).

Bei der Beschreibung von verschiedenen griechischen Dialekten wurde die lexikalische Ebene oft beiseite gelassen. Das lexikalische Material, das in den epigraphischen Quellen, in der Anthroponymie und in den Glossen vorliegt, hat kaum die ihm gebührende Beachtung gefunden. Das liegt zum Teil daran, daß die meisten der in den Inschriften belegten Formen nur als "normale griechische Wörter" zu betrachten sind, bei denen nur die Phonetik und seltener die Morphologie ihren dialektalen Charakter erkennen läßt. Es scheint aber, daß das Studium der dialektalen Lexik speziell zur Erläuterung der interdialektalen Beziehungen und sogar der Herkunft der griechischen Dialekte beitragen kann,

1 Vgl. die fragmentarische Evidenz der in Naukratis im Nildelta gefundenen Keramik.

EMERITA (EM) LXXIV 2, julio-diciembre $2006 \quad$ pp. 233-258 ISSN 0013-6662 
vgl. hierzu die Kriterien bei García Ramón 1997, S. 522ff.:

a) die Form ist in gleicher Bedeutung in anderen Dialekten belegt und auch im Attischen und in der кovví (d.h. gemeingriechisch)

b) die Form gehört der кovví an, d.h. hat dialektales Kolorit (Orthographie, Phonetik, Morphologie)

c) die Form ist in anderen Dialekten belegt, nicht aber im Attischen, bzw. in der кoเví

d) die Form kommt nur in einem Dialekt vor, nicht aber im Attischen, bzw. in der кoเvท́

e) die Form ist ausschließlich im Dialekt einer bestimmten Region belegt, ohne Entsprechungen in anderen Dialekten zu haben

f) die Form ist spezifisch/charakteristisch für einen Dialekt und steht den Formen in anderen Dialekten entgegen.

Zur Ausarbeitung des dialektalen Wortschatzes wurden von Hodot 1987 und García Ramón 1997 zwei methodologische Modelle propagiert. Es wurde zwar teilweise die Lexik des Arkadischen, Kretischen, Ostjonischen und Thessalischen bearbeitet, es fehlt jedoch bis heute für jeden der griechischen Dialekte eine systematische, monographische Darstellung des Wortschatzes. Es fehlt überdies ein dialektales Wörterbuch, in dem man Synonyme der anderen Dialekten finden könnte. Auch das Material der dialektalen Glossen wurde nie kritisch bearbeitet und mit den epigraphischen Daten von bestimmten Dialekten verglichen.

In seinen Untersuchungen zum thessalischen Wortschatz schlägt García Ramón folgende Fälle vor, mit denen man rechnen müsse, wenn man sich mit dem dialektalen Wortschatz befaßt:

1. verschiedene Formen für den gleichen (gemeingriechischen) Inhalt,

2. gleiche Form mit verschiedenen Bedeutungen, vgl. $\pi$ aıs in lesbischen Inschriften

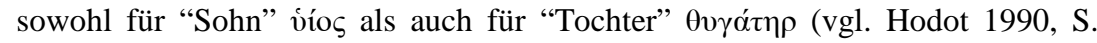
229ff.).

3. die Existenz von Wortbildungsvarianten,

4. termini technici, hapax legomena, Namen von Sozialinstitutionen,

5. hyperkorrekte Formen (Hyperäolismen).

Es scheint, daß man sich bei der Interpretation der Formen dessen bewußt sein muß, daß sich unterschiedliche Konstellationen ergeben können: das lexikalische Material kann unterschiedliche Formen bieten, deren Bedeutung aber übereinstimmt; umgekehrt kann man auch Beispiele finden, in denen formal 
Identisches mit unterschiedlicher Bedeutung belegt ist. Die Formen können zudem in unterschiedlichen Wortbildungsvarianten bezeugt sein. Eine große Gruppe von ihnen bilden spezielle Termini, die sich auf soziale Einrichtungen beziehen. Ferner scheint es, daß die termini technici und hapax legomena einer besondere Behandlung bedürfen, ebenso wie die hyperkorrekten Wörter, die in Übereinstimmung mit dem Charakter der Inschrift und unter Berücksichtigung der sich für diese Epoche ergebenden spezifischen Probleme, z.B. der archaisierenden Tendenzen im 2. Jh. n.Chr. auf Lesbos, gedeutet werden sollten. Das Interpretationsschema sollte immer folgende Information enthalten: a) graphische Varianten und - wenn nötig - eine Diskussion der Orthographie (lectio$n e s)$; b) eine Wortbildungsanalyse c) eine Bedeutungsanalyse: die innerlesbischen Synonyme, die Beziehung zu den anderen Dialekten: кovvท́ : hybride Formen (besonders in hellenistischen Dokumenten und in den Inschriften aus der Kaiserzeit, schließlich e) wenn möglich, die Wortgeschichte.

Das Lexikon der lesbischen Inschriften ist nicht homogen. Das epigraphische Material kann vom semantischen Standpunkt folgendermaßen interpretiert werden, z. B.:

\section{Wirtschaftliches Vokabular}

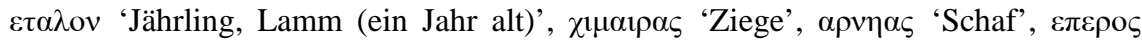
'Widder', $\varepsilon \rho \sigma \varepsilon v$ 'Männchen, Bulle, Hammel', $\pi \varepsilon \pi \alpha \mu \varepsilon v o \varsigma$ 'der Besitzende', $\pi \varepsilon \rho v \alpha \mu \iota$ 'verkaufen'.

\section{Offizielles Vokabular}

$\pi \alpha \sigma v \delta 1 \alpha \zeta \omega$ 'versammeln (Leute)', $\varepsilon \mu \varphi \alpha v i \zeta \omega$ 'ein Manifest veranstalten, proklamie-

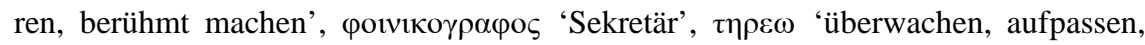
beobachten', $\alpha v \varepsilon \rho \imath \theta \varepsilon v \tau \omega \varsigma$ 'unbestechlich, unparteiisch', $\psi \alpha \varphi \imath \gamma \xi$ 'Abstimmung'.

\section{Sozialstruktur}

$\chi \varepsilon \lambda \lambda \eta \sigma \tau v \varsigma, \chi \varepsilon \lambda \lambda \eta \sigma \tau v \alpha \rho \chi \alpha \varsigma$ 'Tausendschaft, ein Stamm; Führer von ...', $\alpha v \tau \imath \pi \alpha 1 \delta o v o-$

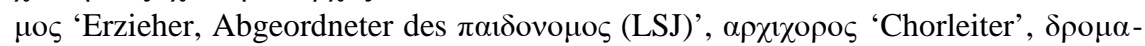
$\gamma \varepsilon \tau \varepsilon \omega$ 'als Beamter bei einem Wettlauf fungieren', $\chi \rho \varepsilon о \varphi v \lambda \alpha \xi$ 'Verwalter des Registers öffentlicher Schuldnern'.

\section{Gericht}

$\alpha v \theta \imath \varepsilon \varepsilon \lambda \eta \varsigma$ 'am Ort und Stelle beendet', $\alpha \gamma \rho \varepsilon \omega, \alpha \gamma \rho \eta \mu \mathrm{t}$ 'nehmen, ergreifen, verurtei-

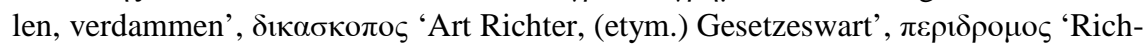
ter (LSJ), Gerichtsbeamter'.

\section{Religion, Kult}

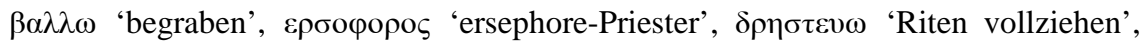

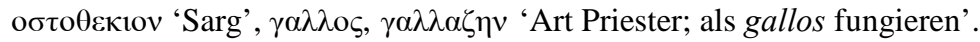

EMERITA (EM) LXXIV 2, julio-diciembre $2006 \quad$ pp. 233-258 ISSN 0013-6662 


\section{Varia}

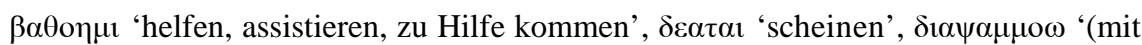

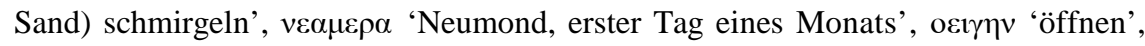
$\sigma \tau \varepsilon \imath \omega \omega$ 'gehen, eintreten'.

Unter den bezeugten Formen können unterschiedliche Kategorien erscheinen:

Typ A: normale griechische Wörter, die im Attischen und in der кovv́ bezeugt sind:

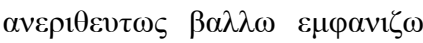

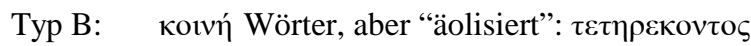

Typ C: Wörter mit Entsprechungen in anderen Dialekten, aber weder im Atti-

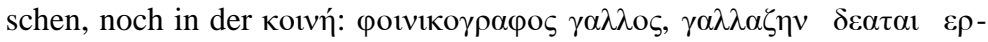

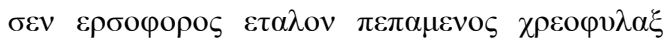

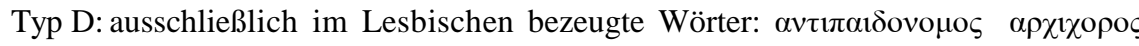

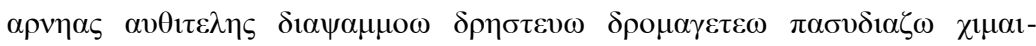
$\alpha$ s

Typ E: $\quad$ spezifische dialektale Termini, die in Opposition zu ihren Synonymen in

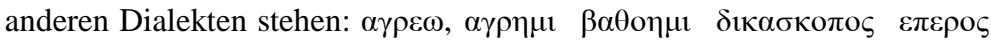

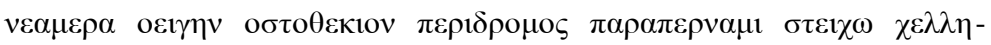
$\sigma \tau v \varsigma \psi \alpha \varphi \imath \gamma \xi$

Im folgenden möchte ich einige interessante in lesbischen Inschriften bezeugte Formen interpretieren. Sie unterscheiden sich in einigen Merkmalen von den entsprechenden attischen Formen, nicht alle sind indessen rein dialektal.

* $\beta \alpha \lambda \lambda \omega \sim$ Att. $\theta \alpha \dot{\pi} \tau \tau \omega$ - 'begraben'

IG XII, 2.406, 4 (Mytilene; wahrsch. 3. Jh. n.Chr.; Grabinschrift)

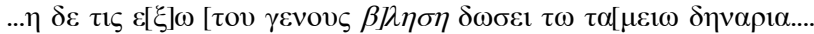

“... wenn jemand nicht von der Familie (begraben wird), soll er dem Fiskus ... Denare zahlen ...”

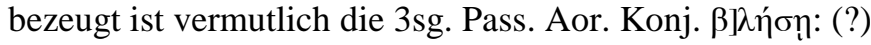

Das Verb $\beta \alpha ́ \lambda \lambda \omega$ scheint in dieser Bedeutung nur in späten Inschriften aus Kleinasien bezeugt zu sein, vgl. Acmonia 3. Jh. n.Chr., MAMA VI 325.15: $\mu \varepsilon \tau \alpha$

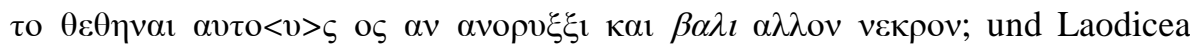

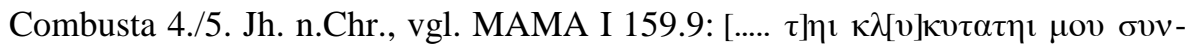

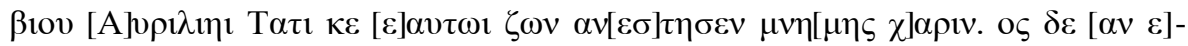

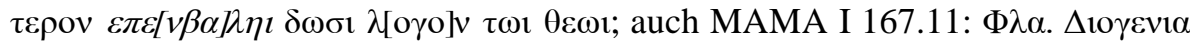

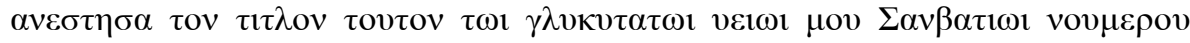

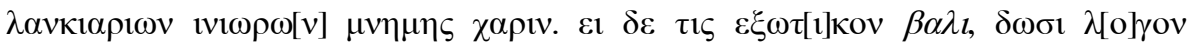

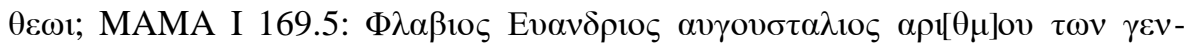

EMERITA (EM) LXXIV 2, julio-diciembre $2006 \quad$ pp. 233-258 ISSN 0013-6662 


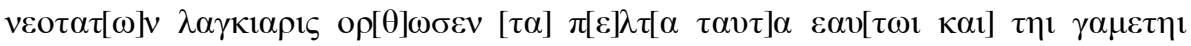

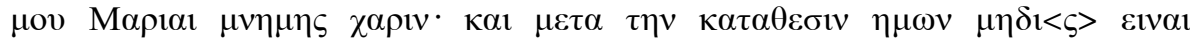

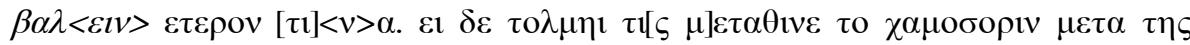

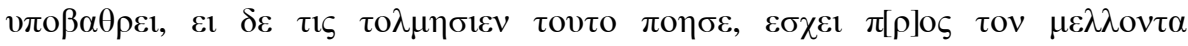
$\kappa \rho \mathrm{iv}<\varepsilon \mathrm{iv}>\zeta \omega v \tau \alpha \varsigma \kappa \alpha \mathrm{l} v \varepsilon \kappa \rho о \nu \varsigma$.

Es dürften im Hinblick auf die Evidenz der lesbischen Steininschriften einige Bemerkungen zur Orthographie erforderlich sein. Das Hauptproblem besteht in der Erklärung der lectio $\mathrm{B}] \Lambda \mathrm{HCH}-$ mit $\langle\Sigma\rangle$ oder $\langle\Theta>$ ? Es ist zu beachten, daß die Möglichkeit einer inverse Schreibung besteht, der Name des Stifters der

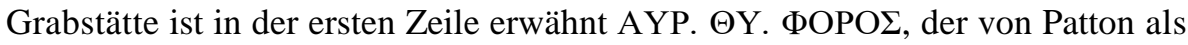
AYPE $\Lambda$ IO $\Sigma$ $\Sigma$ YMФОРО $\Sigma$ transkribiert wurde.

Es erscheint möglich, daß die bezeugte Orthographie im Zusammenhang mit Beziehung zwischen /s/ und /th/ in der altgriechischen Phonologie erklärt werden kann. Nach Allen konnte der mutmaßliche Wandel der aspirierten Klusile zu Reibelauten im 2. Jh. v.Chr. (Allen 1968, 20f.) erfolgt sein. Es ist jedoch das Zeugnis des Aristophanes, der $\langle\sigma\rangle(/ \mathrm{s} /)$ anstelle von attischem $\langle\theta\rangle\left(/ \mathrm{t}^{\mathrm{h}} /\right)$ in Beispielen für den lakonischen Dialekt bietet, z.B.. Ach. 905 Neì $\tau \dot{\omega} \sigma \iota \omega$ (att. $\theta \varepsilon \dot{)}$ ),

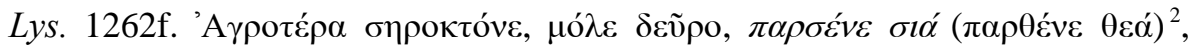

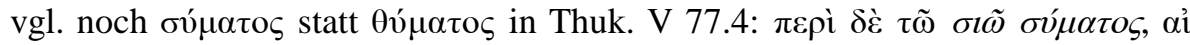

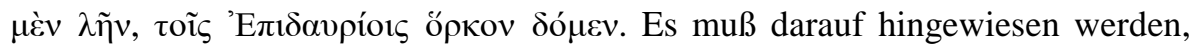
daß eine solche Schreibung auch im lakonischen epigraphischen Material erscheint, vgl. Sparta 4. Jh. v.Chr. IG V 1.785.1: A $\alpha \alpha \sigma ı v$, oder 1115, A col I 31:

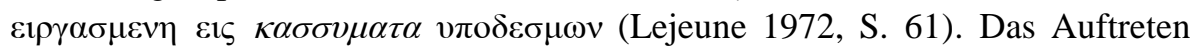
von /s/ statt einer Aspirata wurde auch von den antiken Grammatikern als ein

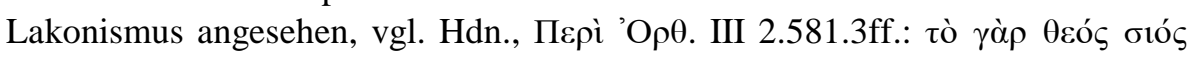

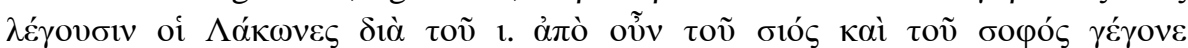

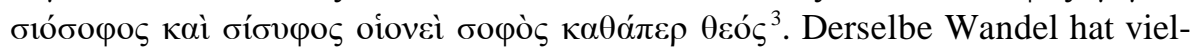
leicht auch in anderen Gebieten Griechenlands stattgefunden, vgl. den PN A $\sigma \alpha \mu \beta$ o $\varsigma$ als Entsprechung zu A $\theta \alpha \mu \beta$ o aus Delphi (Thumb-Kieckers 1932, S. 157), vgl. auch die Beispiele aus dem Attischen gegen Ende des 5. und im 4. Jh.

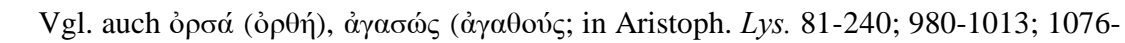
$1188 ; 1242-1321)$.

3 Ein ähnliche Erscheinung findet sich mehrmals bei Alkman, cf. e.g. Fr. 26, 1: oú $\mu^{\prime}$

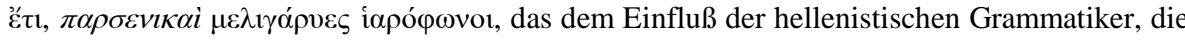
die Texte herausgaben, zugeschrieben wurde; das Problem ist «ob $\sigma$ in diesen Fällen einen Dental $[\theta]$ vertritt oder ob es sich in Lakonien schon zu alveolarem $[\sigma]$, das in seinem modernen Fortsetzer, dem Tsakonischen, bezeugt ist, gewandelt hat» (Thumb-Kieckers 1932, 79; vgl. auch Allen 1968, 26).
}

EMERITA (EM) LXXIV 2, julio-diciembre $2006 \quad$ pp. 233-258 ISSN 0013-6662 


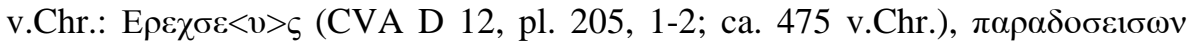

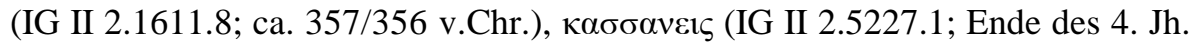
v.Chr.; Teodorsson 1974, S. 134); ebenso in der Ptolemäischen кoเvท́, in der die folgenden Belege zu finden sind: $\mu \varepsilon \sigma 1 \delta$ ov (PCZ I 59044.25; 257 v.Chr.), $\varepsilon \alpha v$

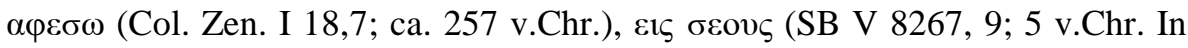
Diesem Beispiel scheint jedoch eher das Ergebnis einer Assimilation von $\varepsilon 15$ $\theta$ cov $\varsigma$ vorzuliegen; Teodorsson 1977, 179). Es ist zu betonen, daß es keine Belege für einen solchen Wandel in äolischen Dialekten gibt.; dieses Merkmal ist für das spätere Griechisch charakteristisch.

Die morphologische Interpretation der Form $\beta \lambda \eta \sigma \eta$ ist problematisch. Die wohl beste Lösung ist, die Form als Beispiel eines passivischen Aorists auf $-\theta \eta$ von $\beta \alpha \dot{\lambda} \lambda \lambda \omega$ (mit $\left./ \mathrm{s} / \mathrm{von} / \mathrm{t}^{\mathrm{h}} /\right)$ zu betrachten, vgl.. das Fehlen von Formen des sigmatischen Aorists bei diesem Verb (Voll- oder Dehnstufe wäre zu erwarten). In einem solchen Fall bedarf die Endung $-\eta$ einer Erklärung: Hodot weist auf die Tatsache hin, daß vom 4. Jh. an die Formen des Konjunktivs Aorist Passiv auf -

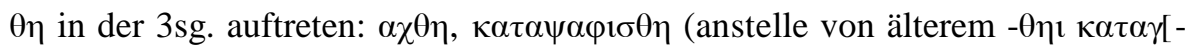
$\rho \varepsilon] \theta \eta \imath)$, solche Varianten entsprechen gewöhnlich bezeugten кoเvฑ-Formen (Hodot, 1990, 203f.), vgl. auch ähnliche Inschriften, die immer den Aor. Konjunktiv bieten, z.B.. IG XII 2.644.10f. (Tenedos) $\varepsilon 1 \mu \varepsilon \tau \alpha \theta \eta, \delta \omega \sigma \varepsilon 1 .$. (vermutlich aus der christlichen Ära); IG XII 2.562.6 (Eresos) $\varepsilon 1 \delta \varepsilon \tau \iota \varsigma \tau 0 \lambda \mu \eta \sigma \eta \varepsilon \pi ı \beta \alpha \lambda \varepsilon \sigma-$ $\theta \alpha \iota \pi \tau \omega \mu \alpha \ldots . ., \delta \omega \sigma \varepsilon 1 \ldots$ ( $\varepsilon \iota \delta \varepsilon-k a n n$ in Konditionalsätzen - außer in attischer Prosa - dem Konjunktiv vorausgehen).

Vielleicht finden sich noch zwei weitere Beispiele in ähnlichem Kontext un-

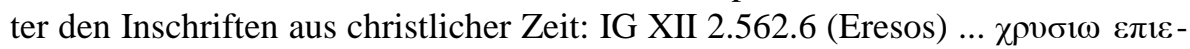

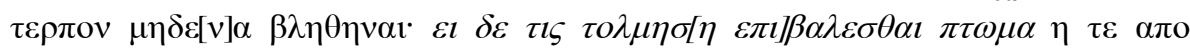

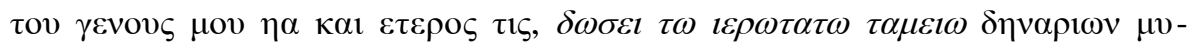
$\rho ı \delta \delta \varsigma$... "wenn sich jemand erdreistet, einen Leichnam niederzulegen, so soll er zehtausende ... Denar zahlen ..."; und IG XII 2.644.10f. (Tenedos) ... \&1 $\tau\rceil \varsigma$

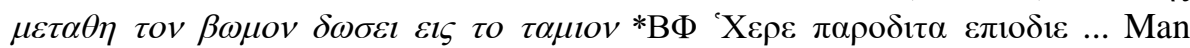
kann die gleiche spezifische Sachlage wie in den $\beta \lambda \eta \sigma \eta$-Inschriften in der Begräbnisformel beobachten, in beiden Fällen ist eine Geldstrafe erwähnt. Es erscheint höchst zweifelhaft, daß $\beta \alpha \alpha \lambda \lambda \omega$ in der Bedeutung 'in die Gruft legen, begraben' gebraucht werden konnte. Im klassischen Griechischen dürfte ein solche Verwendungsweise nicht zu belegen sein. Eine solche Semantik kommt in der späten кoıv́ der Kaiserzeit vor, vgl. «de cadáveres, frec. en epitafios poner ba-

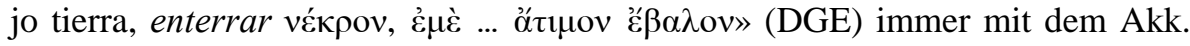
der Person (Objekt); vgl. das schon oben zitierte MAMA VI 325.15: $\beta \alpha \lambda \iota \lambda \lambda$ -

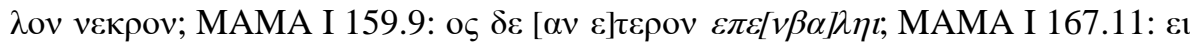

EMERITA (EM) LXXIV 2, julio-diciembre $2006 \quad$ pp. 233-258 ISSN 0013-6662 


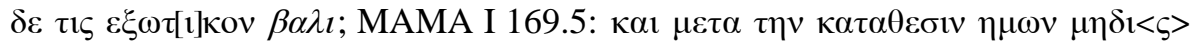
$\varepsilon i v \alpha l \beta \alpha \lambda<\varepsilon ı v>\varepsilon \tau \varepsilon \rho \circ v[\tau 1]<v>\alpha$. Es scheint, daß die in Mytilene bezeugte Form $\beta \lambda \eta \sigma \eta$ ebenfalls zu dieser Gruppe gehört.

Die ganze Inschrift stammt aus einer sehr späten Epoche, das Dokument ist nicht im Dialekt verfaßt, zumindest können keine dialektalen Merkmale beobachtet werden. Nichtsdestoweniger kann man sagen, daß sich typische Merkmale des späten Griechischen finden lassen (der Wandel von $/ \mathrm{th} />/ \mathrm{s} /$ ). Die Bedeutung von $\beta \alpha ́ \lambda \lambda \lambda \omega$ 'begraben' kann ebenso ein Merkmal des Spätgriechischen sein.

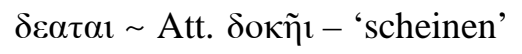

Gauthier 1999, 3 Zeile 17 (AIG 3. Jh. v.Chr.; Ehrendekret)

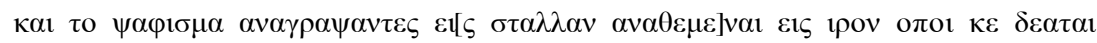
$\varepsilon \pi \imath \tau \alpha \delta \varepsilon \circ \nu \varepsilon \mu \mu \varepsilon v \alpha \imath$

... und als sie das Dekret auf die Säule geschrieben hatten, stellten sie es im Tempel auf, wo es ihnen passend schiene ...

belegt in der 3.sg. Konjunktiv Präsens Medium.

Eine ähnliche Form ist im Arkadischen belegt, vgl. IPArk 3, 10 (Tegea 350

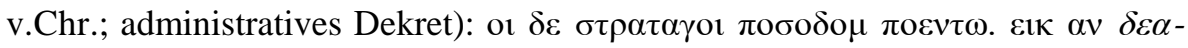

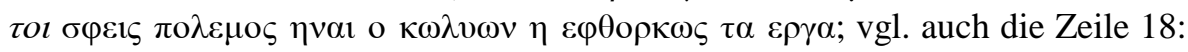

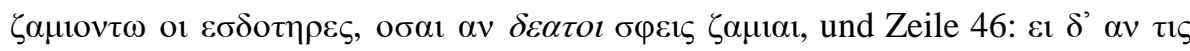
$\tau \omega v \varepsilon \rho \gamma \omega v \alpha v \eta \tau \omega v \varepsilon \rho \gamma \alpha \zeta \circ \mu \varepsilon v \omega v \varepsilon \pi \eta \rho \varepsilon 1 \alpha \zeta \varepsilon v \quad \delta \varepsilon \alpha \tau o l$ iv $\tau \alpha \varepsilon \rho \gamma \alpha \eta$ $\alpha \pi \varepsilon 1 \theta \eta v \alpha$; vgl. auch IPArk 15 A 30 (Orchomenos 360-350 v.Chr.; Dekret betreffs einer synoikia), Aor. Konj.: $\gamma \rho \alpha \varphi \varepsilon \alpha$ $\gamma \rho \alpha \psi \alpha v<\tau>\alpha \varsigma \kappa \alpha \theta[\varepsilon \sigma] \theta[\alpha l]$ o $\pi \mathrm{o} \theta^{\prime} \alpha v \quad \delta \varepsilon \alpha[\sigma \eta] \tau \mathrm{ol}$

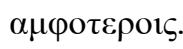

Das Vorkommen der Form $\delta \varepsilon \alpha \tau \alpha \iota$ im epigraphischen Lesbischen scheint unbeschadet seiner spärlichen Rezeption von großer Bedeutung zu sein. Vor der Veröffentlichung der Inschrift von Claros, wurde ein solcher Konjunktiv als eine Besonderheit des arkadischen Dialekts angesehen, die den anderswo be-

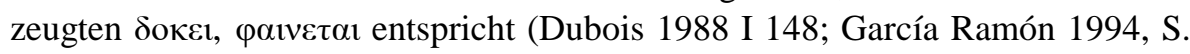

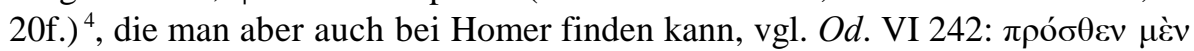

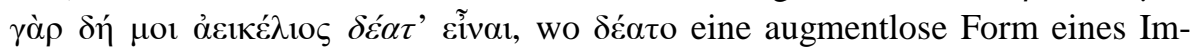
perfekts ist ${ }^{5}$. Bei der Interpretation des lesbischen $\delta \varepsilon \alpha \tau \alpha \iota$ ist die Möglichkeit auszuschließen, daß es sich um einen Homerismus handelt. Im vorliegendem

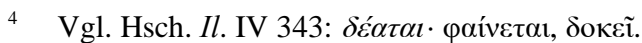

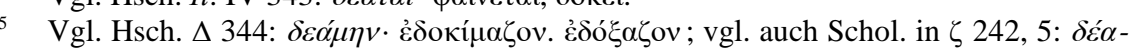

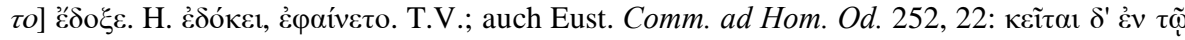

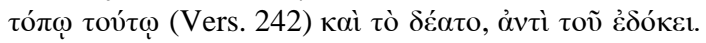

EMERITA (EM) LXXIV 2, julio-diciembre $2006 \quad$ pp. 233-258 ISSN 0013-6662 
Fall kann diese Annahme leicht ausgeschlossen werden, da es sich um kein poetisches Dokument handelt. Die im Jahre 1996 gefundene Inschrift gehört zur Gruppe der Ehrungsdekrete, die für einen fremden Richter galten: die Stadt Aigai entbietet den Richtern von Colophon ihre Ehrenbezeugung. Der Teil der Inschrift, der Aigai betrifft, wurde unter Verwendung eines offiziellen Stils im

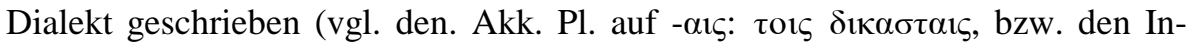
finitiv auf $-\mu \varepsilon v \alpha$, die Pronomina, wie $\alpha \mu \mu \imath$, oder Subst. $\psi \alpha \varphi \imath \sigma \mu \alpha$, usw.), es kommt kein poetisches Vokabular vor (zum Kommentar und den speziellen Beziehungen zu anderen ähnlichen Dokumenten s. Gauthier 1999, 4-11).

$\delta \varepsilon \alpha \tau \alpha_{1}$ setzt ein athematisches Wurzelpräsens voraus *deí $h_{2}$-toÍ < *deí $h_{2^{-}}$'aufleuchten' $\left(\mathrm{LIV}^{2}, 108\right.$, bedauerlicherweise ohne Erwähnung des lesbischen $\delta \varepsilon \alpha-$ $\tau \alpha$ ), die Wurzel ist im Attischen in der Form eines kontrahierten Adjektivs $\delta \tilde{\eta} \lambda$ o $\varsigma$ 'offenbar, deutlich' < *dea-lo- bezeugt (Frisk GEW I Ss. 354, 379; zu seinen Entsprechungen im Vedischen s. García Ramón 1994, S. 21), zum innerem Hiat vgl. ark. Inf.Aor. $\delta \varepsilon F \alpha \lambda \omega \sigma \alpha l$ (IPArk. 9.2; mit historischer Schreibung) ${ }^{6}$, el. ... $\alpha \delta \varepsilon \alpha \lambda \tau \omega h \alpha 1 \varepsilon \ldots$ im Bedeutung 'entfernen' in Olympia (SEG 38, 365, 12). García Ramón weist auf einige Typen von syntaktischen Konstruktionen hin, in denen das homerische $\delta \varepsilon ́ \alpha \tau \alpha$ ( und der Aorist $\delta o \alpha ́ \sigma \sigma \alpha \tau o$ ) vorkommt: a) finit mit nominalem Prädikat; b) impersonal, aber als Formel; c) impersonal mit Infinitiv. Der inschriftlich bezeugte arkadische Konjunktiv kommt in ähnlichen Konstruk-

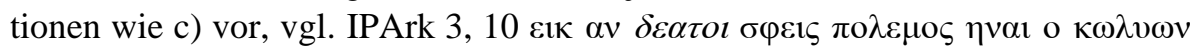

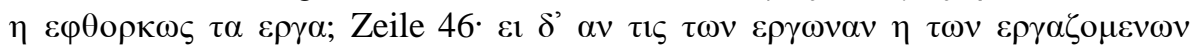
$\varepsilon \pi \eta \rho \varepsilon 1 \alpha \zeta \varepsilon v$ $\delta \varepsilon \alpha \tau o l$ iv $\tau \alpha \varepsilon \rho \gamma \alpha$; ein weiterer Beleg könnte laut García Ramón in IPArk 15 A 30 vorliegen: $\gamma \rho \alpha \varphi \varepsilon \alpha \gamma \rho \rho \psi \alpha v<\tau>\alpha \zeta \kappa \alpha \theta[\varepsilon \sigma] \theta[\alpha 1]$ о оло $\theta^{\prime} \alpha v \delta \varepsilon \alpha[\sigma \eta]-$

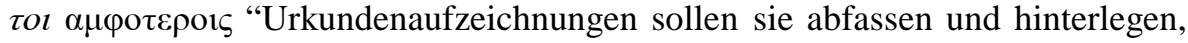
wo immer es beide beschließen" (Thür-Taeuber 1994, S. 132), ein solche Verteilung stimmt mit der von ઠокє̃̃ überein (García Ramón 1994, passim).

Die Bedeutung von $\delta \varepsilon \alpha \tau \alpha \iota$ ist vermutlich gegenüber der ursprünglichen, im vedischen Perfekt dīdāya 'leuchtet' bewahrten Semantik, eine griechische Neuerung (García Ramón 1994, S. 21). Die lesbische Form scheint in funktioneller Hinsicht als eine Art Impersonale mit einem Infinitiv zu Typ c) zu gehö-

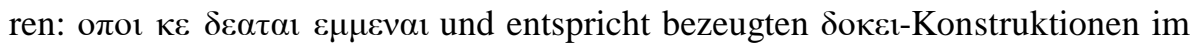
Lesbischen, cf. ERE 01 d 22 (zweite Hälfte des 4.Jh. v.Chr.; politisches Dekret):

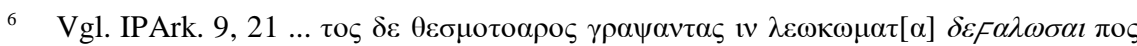

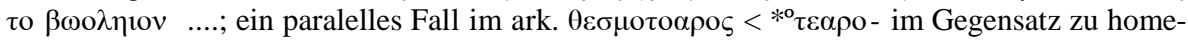
rischem $\tau \tilde{\eta} \rho \varsigma_{\varsigma}$ 'Wächter', Ion-Att. $\tau \eta \rho \varepsilon ́ \omega$ '(be)wachen'.

EMERITA (EM) LXXIV 2, julio-diciembre $2006 \quad$ pp. 233-258 ISSN 0013-6662 


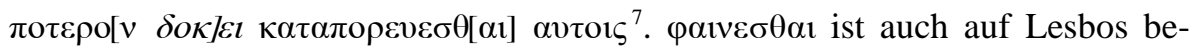

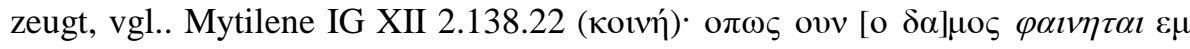

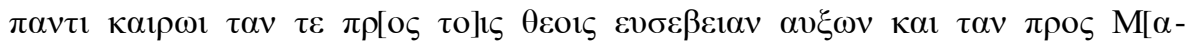

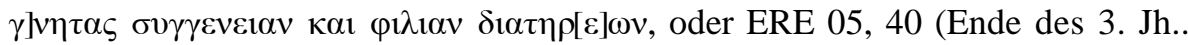

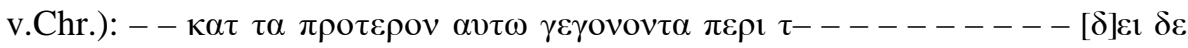

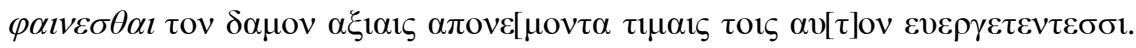

Der Gebrauch von $\delta \varepsilon \alpha \tau \alpha \iota$ im Lesbischen kann als ein spezielles dialektales Merkmal gewertet werden, als ein ererbtes Element im Arkadischen und als ein Archaismus bei Homer. Das Beispiel ist leider im Lesbischen isoliert, aber es mag ein Indiz für die Existenz der Form im volkssprachlichen Gebrauch sein.

ઠъкабколо - - 'eine Art Richter', etym. 'Hüter des Gesetzes'.

MYT 04, 12.14 (ca. 332 v.Chr.; offiziell-politisches Dekret)

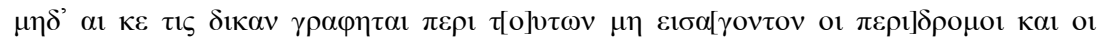

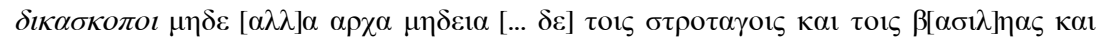

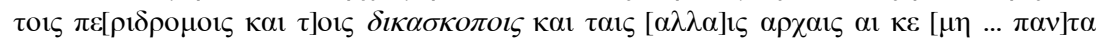

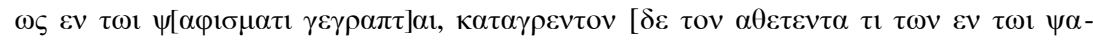
$\varphi 1 \sigma \mu \alpha \tau \imath ~ \gamma \varepsilon \gamma \rho \alpha] \mu \mu \varepsilon v \omega v$.....

if anyone brings in a suit about these matters, neither the peridromoi nor the dikaskopoi nor any other official shall introduce it ... it is to be the responsability of the strategoi and the basileis and the peridromoi and the dikaskopoi and the other officials, if all things do not take place as written in the decree to condemn the one disreading any of the things written in the decree ... (transl. Heisserer 1980, S. 124f.)

KYM 06, 6.7.14. (3. Jh. v.Chr.; offiziell-politisches Dekret)

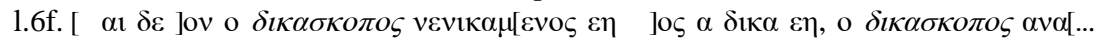
...(wenn) dikaskopos besiegt wird... ... (wenn) ein Verfahren stattfindet, (soll) der $d i$ kaskopos....

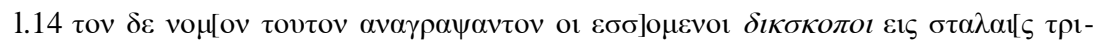

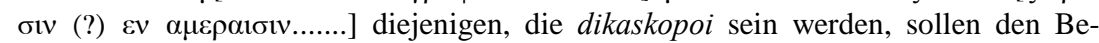
schluß innerhalb von drei Tagen auf die Säulen schreiben ...

Gauthier 1999, 3, Zeile 10 (AIG 3. Jh. v.Chr.; Ehrendekret)

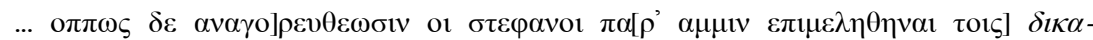
бколог૬ ... "wenn wir die Verleihung von Kronen verkünden, sollen die dikaskopoi Sorge tragen, ..."

bezeugt sind die Formen Nominativ Singular, Nom. Pl. und Akk. Pl..

Der Titel zeugt.

Die Form verursacht hinsichtlich Orthographie und Phonologie keine Probleme. Das Wort ist ein Beispiel für ein mit dem Akkusativ Plural gebildetes

7 Vgl. oft bezeugtes $\varepsilon \delta$ o $\xi \varepsilon$ in lesbischen Inschriften in der Eröffnungsformel des Dokuments.

EMERITA (EM) LXXIV 2, julio-diciembre $2006 \quad$ pp. 233-258 ISSN 0013-6662 
Kompositum mit einem $o$-stufigen Verbalnomen бко́ $\pi$ o 'der aufpaßt, Wächter,

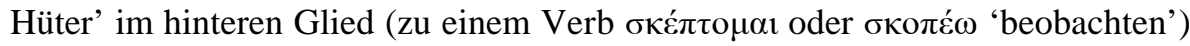

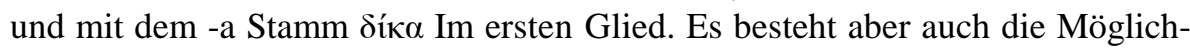
keit, daß es sich um mit dem Akkusativ Plural gebildetes Kompositum mit $\delta$ óka $\varsigma^{\circ}<*$ dikans im ersten Glied handelt ${ }^{8}$. Diese Form scheint analogisch nach

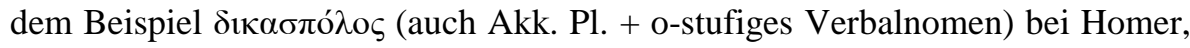
wo es in der Bedeutung 'Richter' vorkommt, gebildet zu sein, vgl. z.B. Il. I 238:

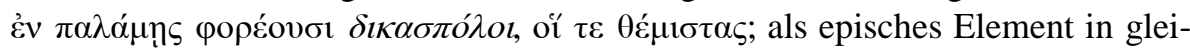
cher Bedeutung auch in der Posthomerischen Dichtung, z.B. A.R. 4, 1178

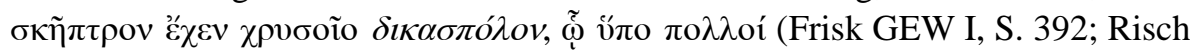
1974, S. 220) ${ }^{9}$.

Die semantische Bewertung der Form gestaltet sich problematisch. Dem Kontext beider Inschriften können wir entnehmen, daß die dikaskopoi öffentliche Beamte waren, die darauf zu achten hatten, daß das Gesetz befolgt wird. Sie

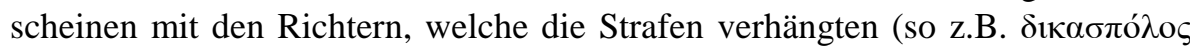
bei Homer) und die auf Lesbos wie in den anderen Gebieten Griechenlands $\delta$ $\kappa \alpha \sigma \tau \alpha$ genannt wurden, nicht identisch gewesen zu sein. In KYM 06, 8 findet

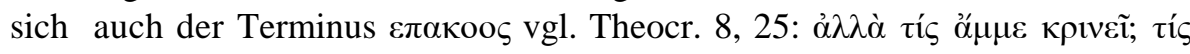

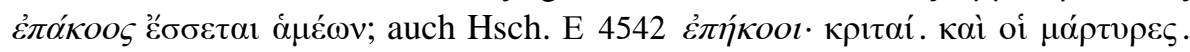

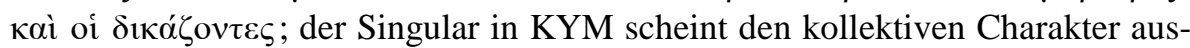
zudrücken (vgl. Engelmann 1976, 18). Eine vermutlich parallele Bildung findet sich im Arkadischen, wo ein Nomen $\theta \varepsilon \sigma \mu о \tau$ o $\rho \rho \varsigma$ vorkommt, cf. Mantineia

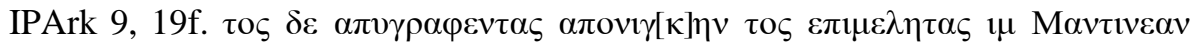

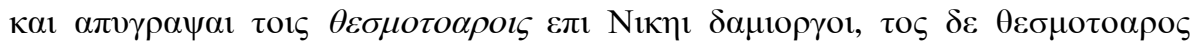

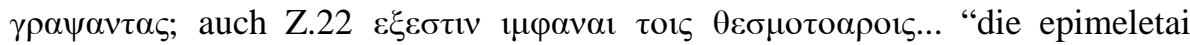
sollen die Liste der Registrierten nach Mantineia bringen und diese bei den Thesmotoaroi unter dem Damiorgos Nikes registrieren"; Z.22 "(er) soll fähig sein den Thesmotoarois zu zeigen". Das arkadische $\theta \varepsilon \sigma \mu o \tau$ o $\rho \circ \varsigma_{\text {kann }}$ auch mit 'Hüter des Gesetzes' wiedergegeben werden und ist ebenfalls ein Kompositum mit dem nominalen ersten Element $\theta \varepsilon \sigma \mu o ́ s$, wogegen das zweite auf ein ${ }^{* \circ} \tau \varepsilon \alpha-$

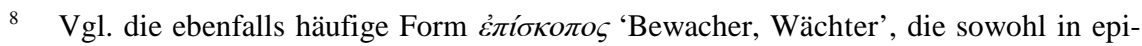
graphischen, als auch in literarischen Quellen unter Einschluß der Dichtung bezeugt ist. Bei Homer ist neben der Bedeutung 'Wächter' auch die Bedeutung 'Kundschafter' zu finden vgl. z.B. in Z 38. Auf Rhodos drückt der Ausdruck eine Art "Magistratsbeamten", in Athen wiederum eine Art "Aufseher, Inspektor" (LSJ) aus.

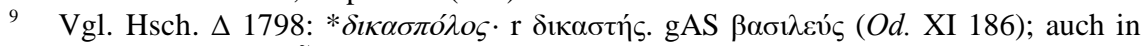

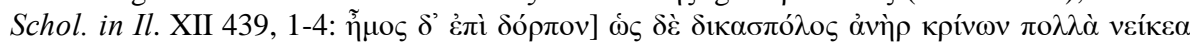

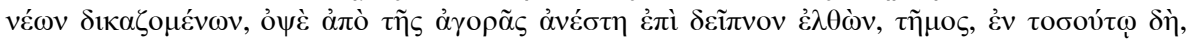

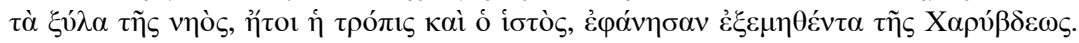

EMERITA (EM) LXXIV 2, julio-diciembre $2006 \quad$ pp. 233-258 ISSN 0013-6662 


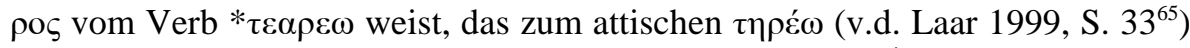
stimmt ${ }^{10}$. Die vorgeschlagene Entwicklung *otea-ros $\left(<* k^{\mathrm{u}} e e^{\prime} h_{2}-r o-\right)>t \bar{e}$ hat in ark. $\delta \varepsilon \alpha \tau o l$ (<*deí $h_{2^{-}}$'aufleuchten', cf. LIV $\left.{ }^{2}, 108\right)$, hom. $\delta \varepsilon ́ \alpha \tau \alpha 1$, lesb. $\delta \varepsilon \alpha \tau \alpha 1$ (s. oben) gegenüber jon.-att. $\delta \tilde{\eta}-\lambda \mathrm{o} \varsigma$ eine Parallele ${ }^{11}$. Nach Waanders kann die $o$ Stufe in ${ }^{\circ} \tau o \alpha \rho \circ \varsigma$ als analogisch nach den anderen Komposita des Typs $\delta 1 \kappa \alpha \sigma-$ ко́ло (mit $o$-Stufe im Zweitglied, Waanders 1987, S. 189) erklärt werden. Ferner scheinen im Falle der thesmotoaroi die für die genaue Bestimmung ihres Aufgabenkreises nötigen Angaben zu fehlen. Offenbar haben sie aber eine bedeutende Rolle in der Stadt gespielt: sie waren für die Vorbereitung der Liste der neuen Bürger verantwortlich. Des weiteren scheint ein $\theta \varepsilon \sigma \mu о \tau$ o $\rho \rho \varsigma$ Art Staatsanwalt gewesen zu sein, welche die Prozesse in die Wege zu leiten hatten (cf. Thür-Taeuber 1994, S. 105f.). Bei den arkadischen thesmotoaroi haben wir es mit einer offiziellen Kongregation zu tun, während die lesbischen ठıкабколо eher als eine den attischen "Sykophanten" ähnliche Einrichtung betrachtet werden können ("jenes öffentliche Aufpassertum"), und zwar wahrscheinlich im Verein mit der Funktion eines öffentlichen Anklägers in Prozessen (Engelmann 1976, S. 18; Gauthier 1999, S. 9).

Offensichtlich entsprechen die in Mytilene und Kyme bezeugten den in verschiedenen anderen griechischen Dialekten und literarischen Quellen bezeugten Formen, z.B. vo $\mu \mathrm{o} / \theta \varepsilon \sigma \mu \mathrm{o}-\varphi v \lambda \alpha \xi$, $\tau \varepsilon \theta \mu \mathrm{\rho} \varphi \nu \lambda \alpha \xi, \theta \varepsilon \mu 1 \sigma \kappa о \pi \mathrm{o} \varsigma^{12}$; die allesamt die wörtliche Bedeutung 'Gesetzeshüter' bieten (Thür-Taeuber 1994, S. 104). Doch scheint es nicht möglich zu sein, eine Antwort auf die Frage, ob ihre Pflichten mit denen der schen vor) identisch waren, zu geben. Die in Mytilene und Kyme bezeugte

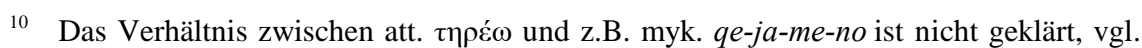
PY Eb 294.1; Ep 704.1; KN X 8532.1. bezeugte qe-ja-me-no / $\mathrm{k}^{\mathrm{w}} \mathrm{ei}(\mathrm{i}) \mathrm{imenos} /$, der als Aor. Med. angesehen werden kann; Die Bedeutung ist unklar («probably a religious title - "reverend”»), die Form hat keine Paralellen in klassischen Griechischen, vgl. Ruijgh 1967, 376.

11 Vgl. auch Dubois, 1988, I, S. 148.

12 Vgl. lesb. MYT 244, 6ff. (3. Jh. n.Chr.; Stiftung): $\varepsilon \pi 1 \tau \varepsilon \tau \varepsilon \lambda \varepsilon \kappa o v \tau \alpha, \kappa \alpha \iota ~ \alpha v \tau o[v] \delta \varepsilon \kappa \alpha 1$

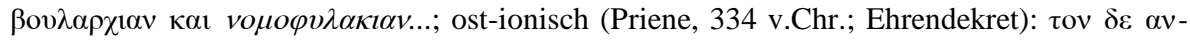

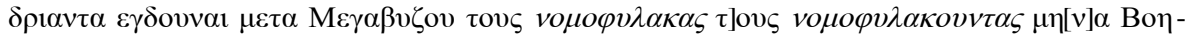
$\delta \rho о \mu \iota \omega[v \alpha \kappa \alpha$... (Hernández Vázquez 1994, S. 449); boeotisch (Orchomenos IG VII, 3172,

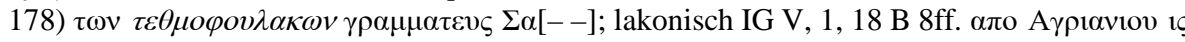

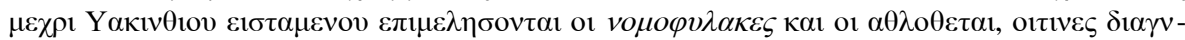

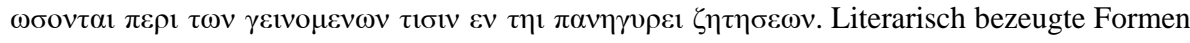

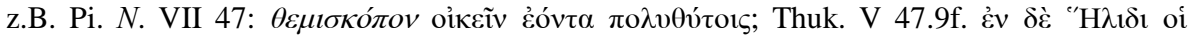

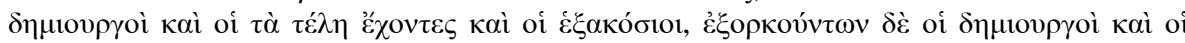

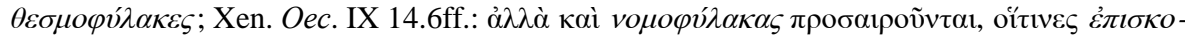

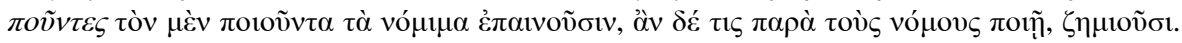

EMERITA (EM) LXXIV 2, julio-diciembre $2006 \quad$ pp. 233-258 ISSN 0013-6662 
Form trägt nichts zur Kenntnis der dialektalen Grammatik bei. Sie entbehrt der phonologischen und morphologischen Merkmale, die für den lesbischen Dialekt kennzeichnend sind. Wenn aber die oben zitierten Formen $\theta \varepsilon \sigma \mu \mathrm{o} \tau$ o $\rho \rho \varsigma$,

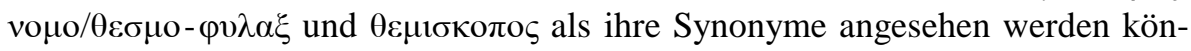
nen, dann dürfte $\delta$ $к \sigma \kappa \kappa \pi о \varsigma$ als ein spezifisches dialektalisches Element und zwar als ein Bestandteil des juristischen Wortschatzes zu deuten sein.

Leider können im Falle von dialektalen Glossen die oben erwähnten Kriterien nicht angewendet werden. Wir können auch feststellen, daß dieses Material nicht ganz zu den Daten paßt, die von den epigraphischen Quellen geboten werden. Was die Herkunft der lesbischen Glossen betrifft, entstammen sie oft poetischen Werken, deren Sprache stark durch homerische Züge geprägt ist. Das Ziel der Untersuchung wäre dann, eine Antwort auf folgende Fragen zu suchen, nämlich ob die Form: Homerisch? poetisch-lesbisch? oder "echt dialektal" (volkssprachig) sein kann.

Das spätestens ins 5./6. Jhd. zu datierende Lexikon von Hesych wurde mit

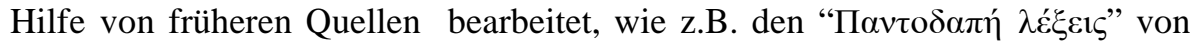

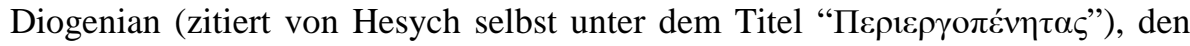
Glossen von Aristarch, Apion, Heliodor und den orthographischen Schriften von Herodian. Die älteste Ausgabe, über die wir verfügen, stammt aus dem 15. Jhd. (Codex Marcianus Graecus 622). Das Lexikon von Hesych hat für die Exegese der klassischen Texte und für die Geschichte des Griechischen großen Wert, es bleibt jedoch eine Frage offen, und zwar die, ob die überlieferten Formen eine zuverlässige Quelle und eine Hilfe bei der Bearbeitung des dialektalen Wortschatzes sein können und speziell, inwiefern sie als Belege für die gesprochene Sprache verschiedener Regionen Griechenlands gewertet werden können.

Die sog. lesbischen Glossen wurden 1893 von Otto Hoffmann zu einem Corpus von ca. 200 Wörtern zusammengefaßt (Die Griechischen Dialekte in ihrem historischen Zusammenhange mit den wichtigsten ihrer Quellen. 2. Band. Der nord-achäische Dialekt; 228-248). Die Hoffmansche Liste umfaßt nur Formen, meistens ohne irgendwelchen Kommentar. Diese Liste bildet den Ausgangspunkt für meine Untersuchung. Jede Form wird als ein einzelner Artikel dargestellt und umfaßt folgende Abschnitte:

In dem ersten werden die Quellen nach folgender Ordnung besprochen:

1. Hesych: mit oder ohne Angabe der dialektalen Zuordnung;

2. andere Quellen (Stellung in der Hoffmannschen Liste): mit oder ohne Angabe;

3. Sekundärquellen: Scholien, Grammatiker;

4. Versuch der Identifikation der ursprünglichen Quellen, aus denen Hesych ge-

EMERITA (EM) LXXIV 2, julio-diciembre $2006 \quad$ pp. 233-258 ISSN 0013-6662 
schöpft hat.

In dem zweiten Abschnitt ist vor allem zu zeigen, ob die Form sonst im lesbischen inschriftlichen Material, bzw. in der lesbischer Lyrik vorkommt; wenn nicht, ob sie bei Homer, oder in der ältesten Dichtung fortlebt; schließlich, ob die Form noch in der кoıví, bzw. in der klassischen Sprache belegt ist; des weiteren, ob eine Form auch in anderen nicht-lesbischen Inschriften (bzw. Dialekten) $\mathrm{zu}$ finden ist.

Im dritten Abschnitt wird die Etymologie zunächst mit Verweis auf das Grundwort bei Ableitungen und die Wortbildung der Glosse besprochen, dann werden die Bedeutungsprobleme (innergriechische Paralellen, bzw. Synonyme) diskutiert; am Ende sollen einzelne Probleme anderer Art (Phonologie, metrischer Einfluß, Textüberlieferung, usw.) erörtert und ein vergleichender Kommentar angeschlossen werden.

Im letzen Abschnitt wird das Fazit der Untersuchung präsentiert, d.h. die Antwort auf die oben gestellten Fragen gegeben.

Das ganze Material kann in drei Gruppen eingeteilt werden. Zur ersten Gruppe sind die Formen, die bei Hesych oder bei anderen antiken Grammatikern als Beispiele der dialektalen Phonetik zitiert werden, zu rechnen, z.B.:

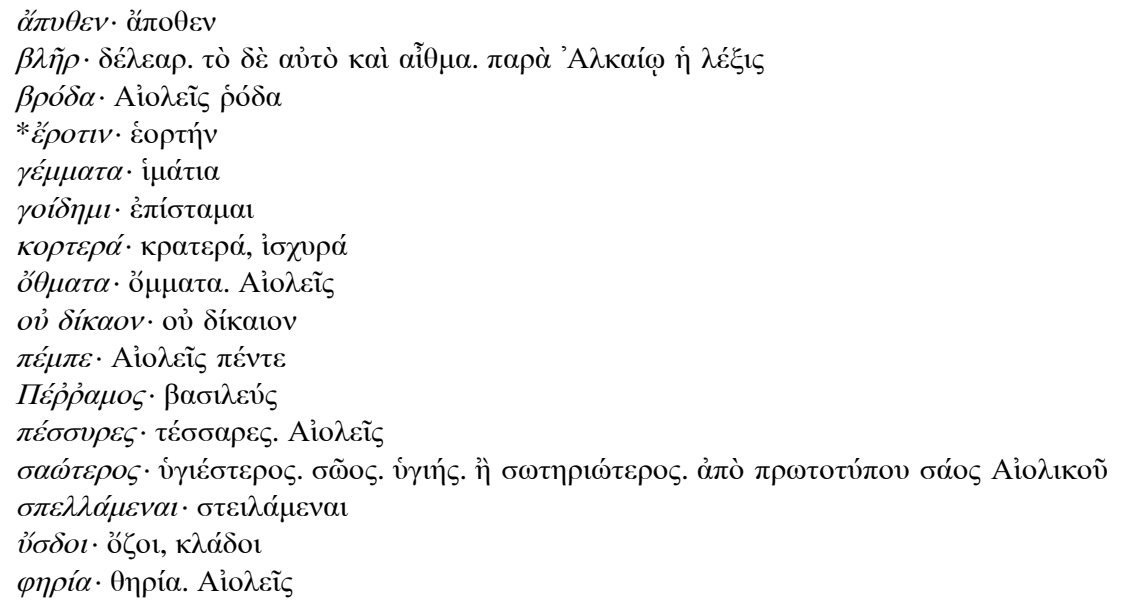

Die zweite Gruppe umfaßt solche Wörter, die morphologische Merkmale aufweisen, z.B.

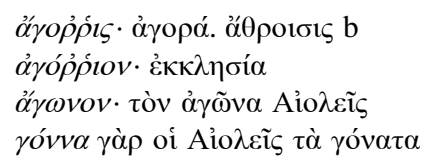

EMERITA (EM) LXXIV 2, julio-diciembre $2006 \quad$ pp. 233-258 ISSN 0013-6662 


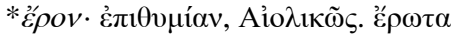

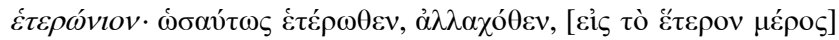
$\mu \varepsilon \mu \beta \lambda \omega v \tau \epsilon v^{\cdot} \tau v \chi o ́ v \tau \omega v$

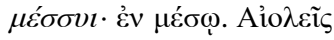
$\pi \varepsilon ́ \rho \sigma \varepsilon \cdot \pi \rho \cos \sigma \varepsilon$

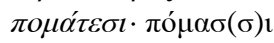

Bei den Formen, die der letzten Gruppe zugewiesen wurden, ergeben sich Probleme aus dem Bereich der Etymologie, der Semantik oder der Wortgeschichte. Diese Gruppe ist die größte, die interessanteste und im Hinblick auf die Bearbeitung die schwierigste. Weil sich die Problematik von einer Form zur anderen ändert, habe ich solche Wörter analysiert, bei deren Interpretation man verschiedene Methoden anwenden kann.

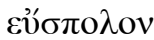

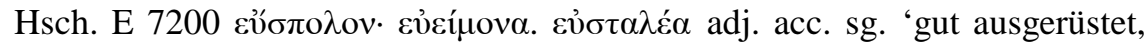
leichtbewaffnet', ohne Hinweis auf eine dialektale Herkunft; in anderen Quellen nicht bezeugt. Die Glosse ist ein hapax legomenon bei Hesych; sie entspricht indessen dem Adjektiv $\varepsilon \dot{\sigma} \sigma \tau \alpha \lambda \eta \dot{\eta}, \varepsilon \varsigma$, das in griechischer Prosa bezeugt ist, und zwar in der Bedeutung 'gut ausgerüstet, (von Truppen) leicht bewaffnet, be-

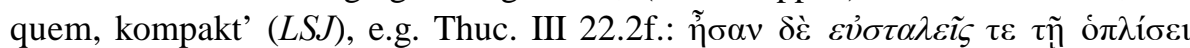

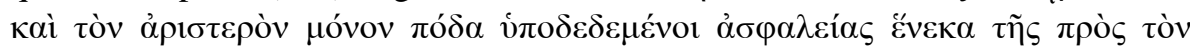

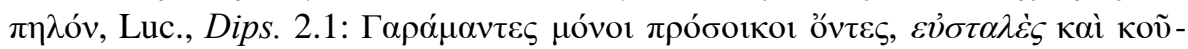

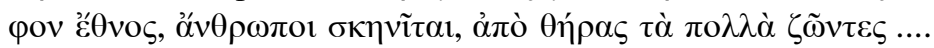

Das Adjektiv scheint im Hinterglied die $o$-stufige Form $\sigma \pi \mathrm{o} \lambda$ - zu enthalten,

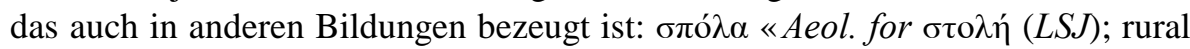

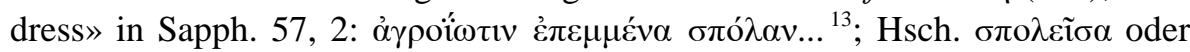
$\kappa \alpha \sigma \pi \mathrm{o} \lambda \dot{\varepsilon} \omega$ (s. unten), Thess. $\sigma \pi \mathrm{o} \lambda \mathrm{o} \varsigma$ 'stake used for palisading' (LSJ).

Es ist verlockend, die Form $\sigma \pi \mathrm{o} \lambda$ - als eine Kontinuante der indogermanischen Wurzel $*_{s} k^{w} e l$ - mit der Bedeutung 'ausrüsten' zu betrachten, wie es

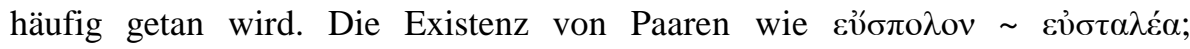

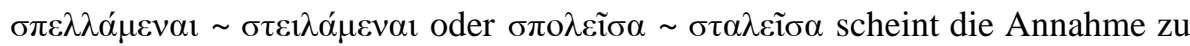
stützen. In allen Fällen können die Formen mit /p/ als Ergebnis einer Verallgemeinerung der o-Stufe $*_{s} k^{w}$ ol- beziehungsweise als Reflex der äolischen Behandlung des ererbten labioverlaren $* / \mathrm{k}^{\mathrm{w}} /$ (d.h. $/ \mathrm{p} /$ vor einem Vordervokal) betrachtet werden (Lejeune 1972, S. 47f.). Diese Rekonstruktion kann zusätzlich

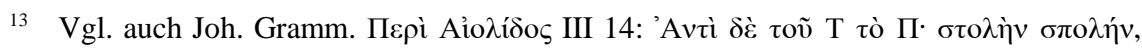
$\sigma \tau \alpha \lambda \varepsilon i \varsigma, \sigma \pi \alpha \lambda \varepsilon i ́$ (Hoffmann 1893, 217).

EMERITA (EM) LXXIV 2, julio-diciembre $2006 \quad$ pp. 233-258 ISSN 0013-6662 
durch das mykenische Nomen qe-ro ${ }_{2} / \mathrm{sk}^{\mathrm{w}}$ ello:/, ein femininer Dual eines unbezeugten *skwella (Derivat in -ía oder -na) 'un corsetto bivalve al cuoio', 'veste protettiva' (del Freo 1996, 81; 83), gestützt werden. Die oben aufgeführten Belege stammen jedoch nur aus grammatischen und literarischen Quellen. Außergriechische Entsprechungen können nicht beigebracht werden, man vergleiche die Rekonstruktion *stel- 'hinstellen, bereit machen' in LIV (mit OCS. po-steljo, -stblati 'ausbreiten, das Bett machen' als das entsprechende -ĺélío- Präsens, LIV 540) ${ }^{14}$. Es ist zu betonen, das das lesbische inschriftliche Material nur die Form $\sigma \tau \varepsilon ́ \lambda \lambda \omega$ liefert, meist mit dem Präverb $\alpha \pi 0^{-}$, die Bedeutung ist nur 'senden' und hat keine Bezeihung zur Semantik 'ausrüsten, bereiten' des mykeni-

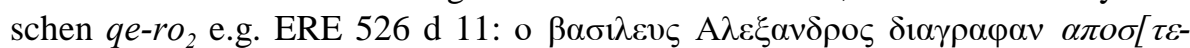

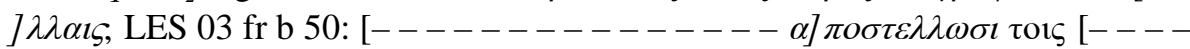

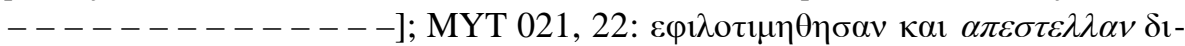

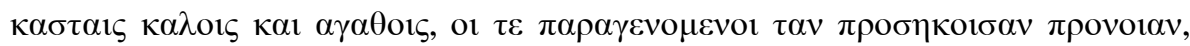
etc. ${ }^{15}$ Eine ähnliche Sachlage findet sich im Thessalischen vgl. z.B. IG IX

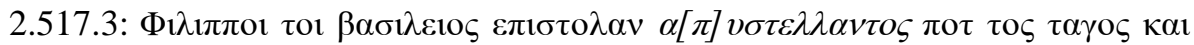

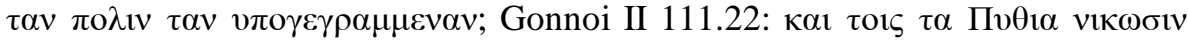

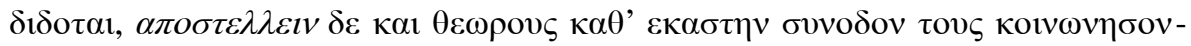

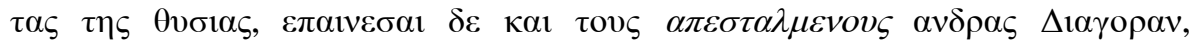

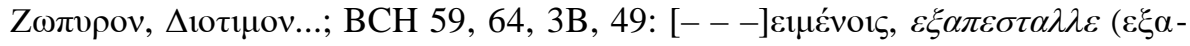
$\pi \varepsilon \sigma \tau \varepsilon \lambda \lambda \varepsilon$ ?) $\delta 1 \kappa \alpha \sigma \tau \alpha \varsigma . .$. . Thessalisch $\sigma \pi \mathrm{o} \lambda \mathrm{o} \varsigma$ 'beim Palisadenbau verwendete Latte' kann als mit $\sigma \tau \mathrm{o} \lambda \mathrm{o} \varsigma$ synonym betrachtet werden, kann jedoch sowohl *sk $k^{\text {e }} l$-, als auch *spel- vertreten (García Ramón 1997, S. 534).

Die Glosse wurde lediglich aufgrund der lautlichen Entsprechung $\sigma \pi-$ : $\sigma \tau$ und wegen $-\mathrm{o} \lambda$ - anstelle von $-\alpha \lambda$ - als lesbisch betrachtet; es ist jedoch festzustellen, $\mathrm{da} \beta / \mathrm{p} /$ vor /o/ im Griechischen zu erwarten ist. Die unmittelbare Quelle der Glosse bleibt im dunkeln; sie fügt sich indessen in den Rahmen der äolischen $\sigma \pi \varepsilon \lambda$-Wörter < $*_{s} k^{w} e l$ - (bezeugt im Mykenischen), die vermutlich von ihren Gegenstücken mit $\sigma \tau \varepsilon \lambda \lambda$-, die sich in anderen Dialekten (und in äolischen Inschriften) belegen lassen, etymologisch nicht identisch sind. Es ist natürlich unmöglich zu entscheiden, ob es sich um ein volkssprachliches oder ein literarisches Element handelt.

14 Hierher vermutlich auch umbrisch ostendu, ustentu 3sg. imp. II < *op-stel-ne-tōd (Meiser 1986, 167f., vgl. Diskussion in WOU 812ff.).

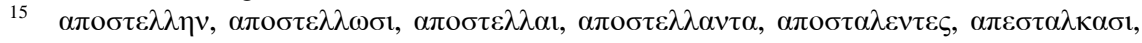
$\alpha \pi \circ \sigma \tau \alpha \lambda \eta \sigma o \mu \varepsilon v 01$.

EMERITA (EM) LXXIV 2, julio-diciembre $2006 \quad$ pp. 233-258 ISSN 0013-6662 
$\kappa \alpha \sigma \pi \varepsilon ́ \lambda \lambda \varepsilon 1, \kappa \alpha \sigma \pi \mathrm{o} \lambda \varepsilon \dot{\varepsilon} \omega$

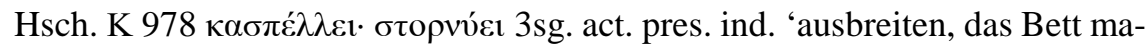
chen'; in anderen Dialekten ist die Form nicht bezeugt. Am nächsten steht hinsichtlich der Bildungsweise der Eintrag K 983 bei Hesych., der die Form der

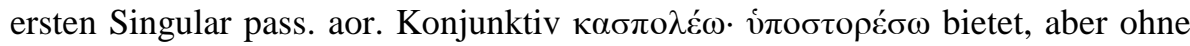
Anzeigen einer dialektalen Herkunft. Die Form ist jedoch bei Herodianus (Pros.

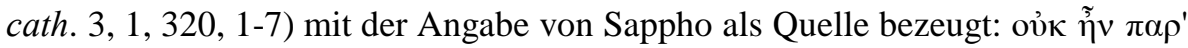

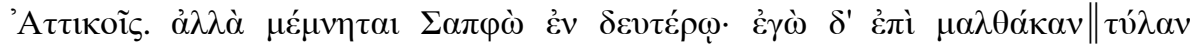

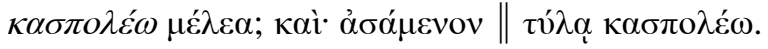

Die Formen sind in lesbischen Inschriften nicht belegbar, $\kappa \alpha \sigma \pi \varepsilon \dot{\lambda} \lambda \varepsilon \varepsilon$ ist ein hapax bei Hesych, andererseits kommt ein $\kappa \alpha \sigma \pi \mathrm{o} \lambda \varepsilon \dot{\omega} \omega$ bei Sappho (vgl. Hamm 1958, S. 145) im Fragment 46 vor:

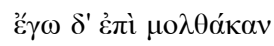

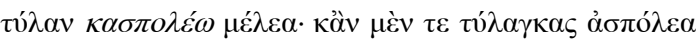

Die Form scheint ein komponiertes Verb zu sein: beim ersten Element handelt es sich um das Präverb $\kappa \alpha \tau \alpha$-, im zweiten Element ist die äolische Wurzel $\sigma \pi \varepsilon \lambda$ -

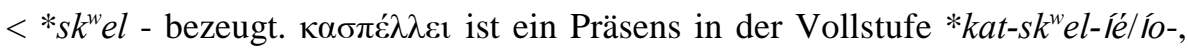
die Form $\kappa \alpha \sigma \pi \mathrm{o} \lambda \varepsilon \dot{\omega} \omega$ sollte als ein unsigmatisches Futur in der Schwundstufe $* k a t-s k^{\mathrm{w}} \notin$, mit der fürs Äolische typischen Behandlung des sonantischen [1] als $o l$ erklärt werden. Die Möglichkeit, dass $\kappa \alpha \sigma \pi \mathrm{o} \lambda \varepsilon \dot{\varepsilon} \omega$ ein denominales Derivat $<* \kappa \alpha \sigma \pi \mathrm{o} \lambda \alpha$ (<*kat-spola) ist, kann als unwahrscheinlich angesehen werden (Rodríguez Somolinos 1998, S. $193^{446}$ ). Beide Formen weisen auf die Existenz der Wurzel *sk $k^{w} e l$ - in der phonetischen Gestalt [spel-], die für das Äolische ty-

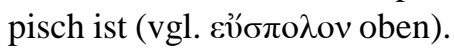

Im ersten Fall bleibt die unmittelbare Quelle im dunkeln, im Falle von $\kappa \alpha \sigma \pi \mathrm{\alpha} \lambda \dot{\varepsilon} \omega$ ist die Quelle mit Sicherheit Sappho. Die Formen dürften literarischer Herkunft sein: es ist dabei beachten, das die einzige Evidenz für äolisch $\sigma \pi \varepsilon \lambda$ - von lesbischen Poeten und antiken Grammatikern geboten wird. Es mag sich um ein archaisches Merkmal in poetischem Gebrauch handeln (vgl. myk. qe-ro 2 ); die volkssprachliche Verwendung, die in den Inschriften bezeugt ist, liefern nur die Formen mit $\sigma \tau \varepsilon \lambda \lambda$-, die Bedeutung ist jedoch immer 'senden' und nicht 'das Bett machen, bereiten'.

$\pi \varepsilon \delta i \tilde{\alpha} \lambda \alpha \iota$

Die bei Hesych in $\Pi 1196$ überlieferte Form $\pi \varepsilon \delta 1 \tilde{\alpha} \lambda \alpha$ im Sinne von

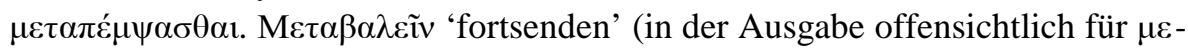
$\tau \alpha \lambda \alpha \beta \varepsilon \tilde{i} v$ der Handschrifte emendiert) kommt nicht in anderen grammatischen

EMERITA (EM) LXXIV 2, julio-diciembre $2006 \quad$ pp. 233-258 ISSN 0013-6662 
Schriften, bzw. Lexika vor. Es fehlen auch irgendwelche Angaben zur möglichen dialektalen Herkunft dieses Wortes.

Man muß betonen, daß die Form bei Hesych als hapax zu betrachten ist. In lesbischen Inschriften gibt es keine Belege von $\pi \varepsilon \delta 1 \tilde{\alpha} \lambda \alpha$, auch die lesbische Dichtung, Homer, die Chorlyrik oder spätere Denkmäler liefern keinen Beleg. Auch die Untersuchung im Rahmen anderer Dialektinschriften bzw. der кovví führt zu einem negativen Ergebnis. Die einzige Evidenz findet sich bei Hesych.

Die Form läßt sich als Aoristinfinitiv erklären und könnte als solche als dialektale Entsprechung des nichtbelegten Kompositums $* \mu \varepsilon \tau 1 \alpha \dot{\lambda} \lambda \lambda \omega$ betrachten werden, mit der äolischen Variante $\pi \varepsilon \delta \alpha$ für $\mu \varepsilon \tau \alpha$ im ersten und dem Verbum $i \alpha \dot{\lambda} \lambda \omega \omega$ 'send forth, put forth, (later) send, dispatch' nach LSJ (vgl. die Definition bei Hesych I $40 * i \alpha ́ \lambda \lambda \omega \cdot \pi \rho \circ \pi \varepsilon ́ \mu \pi \omega$ r ASvg (n)), im zweiten Element. Die Verbalform ió $\lambda \lambda \omega$ selbst ist im Griechischem seit Homer belegt, vgl.: I, 300:

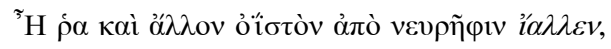

vgl. auch Aor. in $\varphi$ 241:

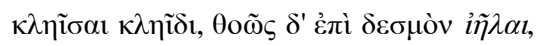

was dem zweiten Element des Kompositums $\pi \varepsilon \delta-1 \tilde{\alpha} \lambda \alpha \iota$ entsprechen kann. Das Wort wurde auch im nachhomerischen Griechisch benutzt, die Evidenz stammt meistens aus der epischen Dichtung, und zwar nur im literarisch-poetischem Kontext ${ }^{16}$.

Was die Wortbildung betrifft, so ist Griechisch iá $\lambda \lambda \omega$ (aor. i ĩ $\lambda \alpha / /$ dor. (Sophron) ió $\lambda \alpha$ ) ein Beispiel für ein redupliziertes Kausativpräsens auf -íélío(Risch 1974, S. 286; mit ganz regulärem Wandel von */li/ > /11/, vgl. Lejeune 1972, S. 171). Die attische aspirierte Variante iá $\lambda \lambda \omega$ ist bei Herodian Pros. cath.

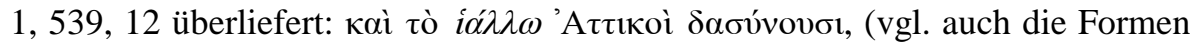

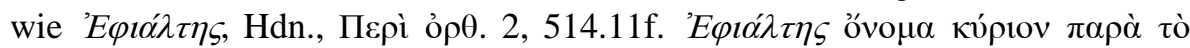

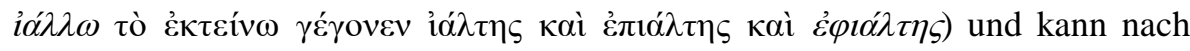
Frisk zu $\alpha \check{\alpha} \lambda \lambda_{\mathrm{o}} \mu \alpha \mathrm{l}$ /hallomai/ gehören, das unter der Annahme einer "Volksetymologie" mit "i $\mu$ verglichen wurde (Frisk GEW 703). Es scheint aber, daß die

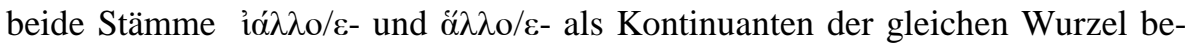
trachten werden können. Lindemann nimmt an, daß Griechisch ió $\lambda \lambda \omega$ als eine athematische deverbative Form mit Vollstufe $* h_{2} i-h_{2} e ́ l-t i$ zu analysieren ist - mit Ved. íyarti 'stößt an' als morphologischer Parallele (<*Hi-Her-ti; Lindemann

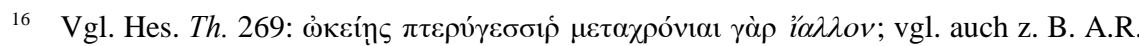

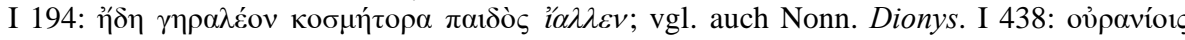

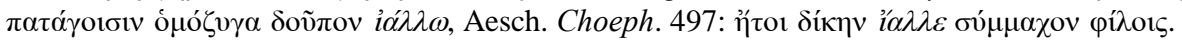

EMERITA (EM) LXXIV 2, julio-diciembre $2006 \quad$ pp. 233-258 ISSN 0013-6662 
1995, 502 ${ }^{14}$ ). Dieser Interpretation steht aber die generell akzeptierte Ansicht

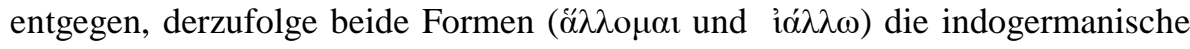
Wurzel *sel- in der Bedeutung 'losschnellen, springen' fortsetzen. ió $\lambda \lambda \omega$ wäre dann ein redupliziertes -íé/ío- Präsens in der Nullstufe *si-sథíe/o- und ebenso

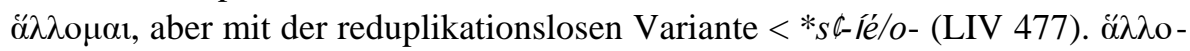

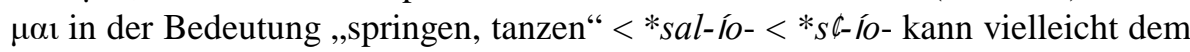
Lateinischem saliō entsprechen (s. LIV 477 für vergleichendes Material; vgl. noch Peters, der ió $\lambda \lambda \omega$ als Fortsetzer von *si-s $\notin$ analysiert 1980, 98).

In lesbischen Inschriften gibt es kein Beispiel für das Vorkommen von ió $\lambda \lambda \omega$ und bei den lesbischen Dichtern ist nur das Verbum ó $\lambda \lambda$ o $\mu \alpha \iota$ vorhanden (vgl. Hamm 1958, S. 134), in einem Fragment, dessen Autor unbekannt ist, vgl. Sappho vel Alc. Fr. 5, 3:

$\varphi \rho \varepsilon ́ v \alpha$

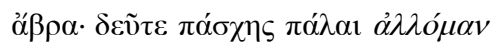

In anderen Regionen von Griechenland kommt ió $\lambda \lambda \omega$ wahrscheinlich einmal inschriftlich vor, und zwar in einem Text aus Smyrna; sollte aber (in der betreffenden poetische Inschrift) eher als epische Form interpretiert werden, vgl. 223, 11 (Hexameter):

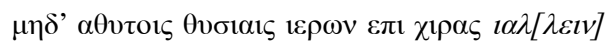

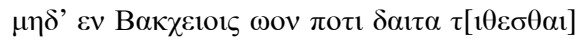

Probleme macht auch das erste Element der Glosse $\pi \varepsilon \delta 1 \tilde{\alpha} \lambda \alpha 1$, die dialektale Präposition $\pi \varepsilon ́ \delta \alpha$ (im Äolischen, Dorischen und Arkadischen) als Entsprechung des ionisch-attischen (auch homerischen) $\mu \varepsilon \tau \alpha$ (Buck 1910, S. 99; vgl. auch He-

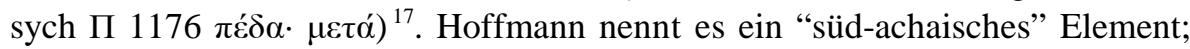

17 Epigraphische Evidenz: Argolis IG IV 552.8: [- $] \tau \varepsilon \varsigma \pi \varepsilon<\delta>\alpha F o \imath \kappa o l \cdot[----]$ Av01$v 0[\varsigma]$; IG IV 615, 1: $\pi \varepsilon \delta \alpha F o<l>\varphi_{O \iota}$ [-]; IG IV 2.1.102 B, 1.97 A

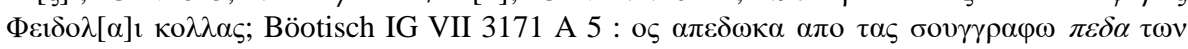

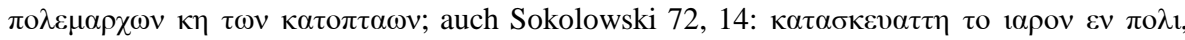
$\beta \omega \lambda \varepsilon v 0 \mu \varepsilon v \omega \varsigma \pi \varepsilon \delta \alpha \tau \omega v \pi \circ \lambda \varepsilon \mu \alpha \rho \chi \omega v \kappa \eta \tau \omega \alpha \rho \chi \imath \tau \varepsilon \kappa \tau o v o \varsigma$. Die Form ist wahrscheinlich auch

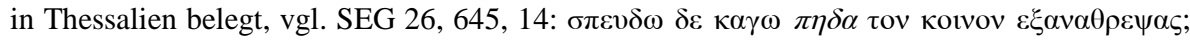
es kommt auf der Dorischen Sporaden vor IG XII 3. 1289.C $1---\kappa \alpha \pi \varepsilon \delta \alpha \mathrm{K}-----$; auf

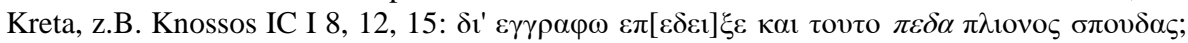

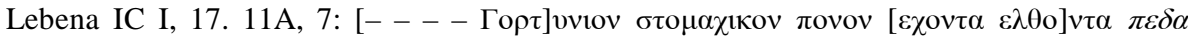

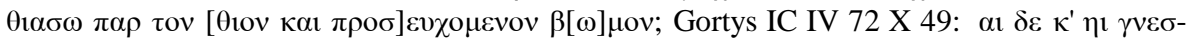

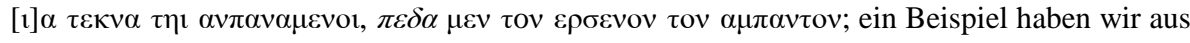

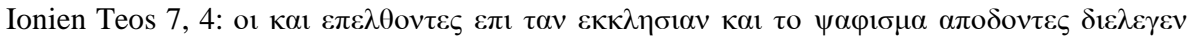

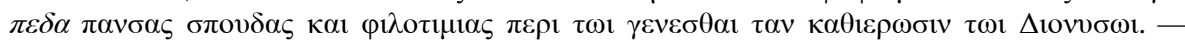
Eine interessante Konstellation kann man in einem Dokument aus Magnesia finden. Beide

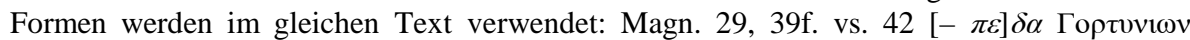

EMERITA (EM) LXXIV 2, julio-diciembre $2006 \quad$ pp. 233-258 ISSN 0013-6662 
seiner Ansicht nach sollte man die Beispiele des dorischen Dialekts mit einem achaischen Substrat auf der Peloponess erklären (Hoffmann 1893, 243). Dieser Ansicht ist auch Schwyzer, der schreibt, daß es sich um ein vordorisches (nicht aber ionisches, auch nicht homerisches) Sprachgut handle (Schwyzer-Debrunner 1950, 499). Die beiden Wörter sind überhaupt nicht verwandt. $\pi \varepsilon \delta \alpha$ scheint zum

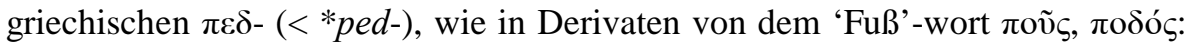
$\delta \alpha ́ \pi \varepsilon \delta \mathrm{ov}, \ddot{\varepsilon} \mu \pi \varepsilon \delta \mathrm{ov}, \pi \varepsilon \zeta$ ó $<*$ pedío- zu gehören (Frisk GEW I 485; nach Meillet BSL 31 Akkusativ Plural mit dem ursprunglichen Sinn: 'auf dem Fuße', mit Armenisch y-et 'nach', $z$-het 'hinterher, nach' zu het 'Fußspur' vergleichbar, Schwyzer-Debrunner, ibidem). Andererseits scheint die Verwendung von $\pi \varepsilon \delta \dot{\alpha}$ und $\mu \varepsilon \tau \alpha ́$ weitgehend identisch zu sein, kleine Unterschiede sind z.B. $\pi \varepsilon \delta \alpha ́$ in der Bedeutung 'inmitten', $\pi \varepsilon \delta \alpha$ als Präfix, keine Beispiele mit Lokativ (vgl.die Funktionen von $\mu \varepsilon \tau \alpha$ bei Schwyzer-Debrunner 1950, 481-87).

Es ist sehr wahrscheinlich, daß sich die Konkurrenz der beiden Wörter noch ins Urgriechische datiert, vgl. myk. me-ta als Präposition in PY An 519.15 meta-qe pe-i, als Präfix in KN Sf. 4428.b me-ta-ke-ku-me-na ${ }^{18}$, PY An 610.3 meta-ki-ti-ta VIR 19 (vgl. DM I 441-46); pe-da in KN Fh 2013 pe-da $a_{\llcorner} i-j e-r o, \mathrm{~V}$ 114b.1 pa-ze a-mi-ni-so pe-da wa-tu /peda unastu/ 'in die Stadt' (Ventris-Chadwick 1973², S. 569; DM II, 94 mit Literatur).

Die Präposition $\pi \varepsilon \delta \alpha ́$ kommt regulär in der lesbischen Dichtung vor, wo keine Belege für $\mu \varepsilon \tau \alpha$ existieren (Hamm, $\left.1958^{2}, 110\right)^{19}$. Es ist in beiden Funktionen belegt: als Präposition und als Präfix in Komposita, vgl. die Beispiele:

Sappho:

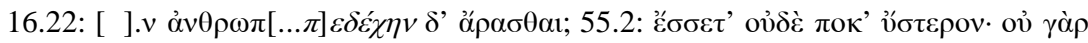

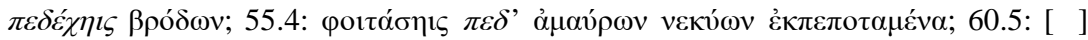

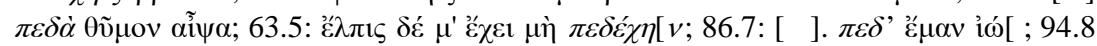

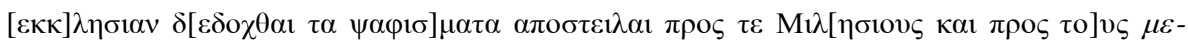

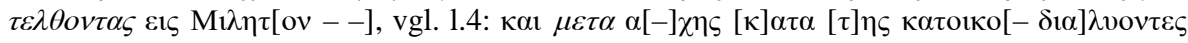
(offentsichtlich wurde der Text zum Teil mit den sprachlichen Zügen der kretischen Gortys abgefaßt).

18 me-ta-ke-ku-me-na KN Sf 4428.b /metak ${ }^{\text {hek }}{ }^{\text {h }}$ Kmena:/ * $\mu \varepsilon \tau \alpha \chi \varepsilon \chi v \mu \varepsilon v \alpha$ (Ruijgh 1967, S. $365)$ oder /metakek umena:/ * $\mu \varepsilon \tau \alpha-\kappa \varepsilon \chi v \mu \varepsilon v \alpha$ (Palmer 1963, S. 434) 'répandu en pièces détachées' (Ruijgh 1967, S. 365). Nom. Sg. Fem. med.-pass. Ptz. Perf. (in Nullstufe) mit dem Präverb me-ta, klass. $\mu \varepsilon \tau \alpha^{-}$, und Form von einem Verbum verwandt mit dem idg. Wurzel *gheút 'gießen', klass. $\chi \varepsilon ́ \omega ~(<U r g r i e c h . ~ * k h e u ́-e l o-)$. Hier im Sinne 'put into pieces', «prefix meta- suggests some form of dismantling or rebuilding» (Ventris-Chadwick 1973², S. 368, vgl. Plath 1994, S. 52 ).

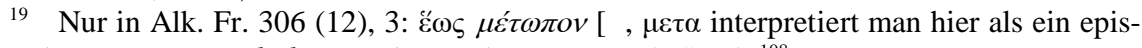
ches Element (Hamm, ibidem, Hodot, nach Hamm 1990, S. $147^{108}$ ).

EMERITA (EM) LXXIV 2, julio-diciembre $2006 \quad$ pp. 233-258 ISSN 0013-6662 


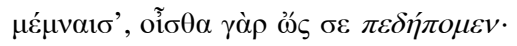

Alkaios:

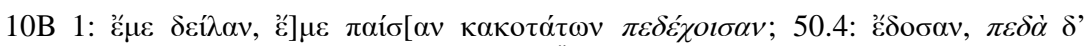

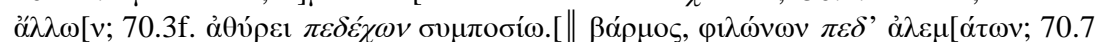

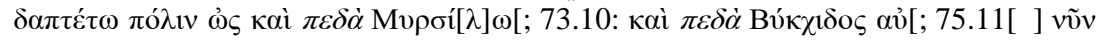

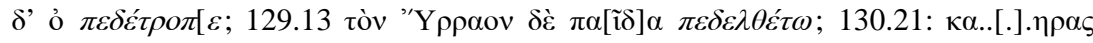

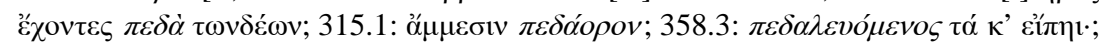

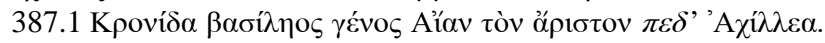

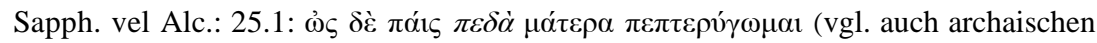

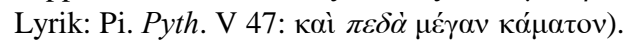

$\pi \varepsilon \delta \alpha$ statt $\mu \varepsilon \tau \alpha$ kommt nur einmal im lesbischen inschriftlichen Material vor, aber sehr früh, d.h am Ende des fünften Jahrhunderts (ca. 426 v.Ch.), vgl. MYT 01, 19-21 (ein politisches Dokument) $\alpha \rho \chi \varepsilon \imath \pi \rho o \tau \alpha v i \varsigma$ o $\pi \varepsilon \delta \alpha$ Ko $\lambda \omega v o v, \varepsilon[\mu$ $\Phi] \omega \kappa \alpha \imath \quad \delta \varepsilon$ о $\pi \varepsilon \delta \alpha$ A $\rho \iota[\tau] \alpha \rho \chi o v$; eine seit dem vierten Jahrhundert belegte Form ist $\mu \varepsilon \tau \alpha$, z.B. ERE 01, a 12 (politisches Text) $\tau \alpha \iota \rho[\alpha] \delta 1 \alpha \rho \pi \alpha \sigma \alpha \iota \varsigma \mu \varepsilon \tau \alpha$ $\tau \omega v[\lambda \alpha] 1 \sigma \tau \alpha \nu$.

Das Vorkommen von $\mu \varepsilon \tau \alpha$ in epigraphischen Quellen im lesbischen Dialekt

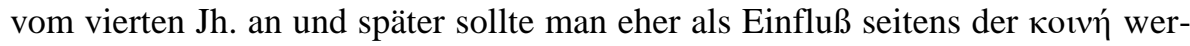
ten. Ingesamt haben wir in epigraphischen Quellen 39 Beispiele als Präposition und ca. 20 als Präfix (Hodot 1990, 147). Eine ähnliche Situation bieten die anderen Dialekte, die normaleweise $\pi \varepsilon \delta \alpha ́$ haben sollten, aber nur Belege mit $\mu \varepsilon \tau \alpha$, «but at the time when кoıv' influence is probable» (Buck 1910, 99) liefern ${ }^{20}$.

Es scheint wahrscheinlich, daß die Glosse $\pi \varepsilon \delta i \tilde{\alpha} \lambda \alpha \iota$ von Hoffmann dem Äo-

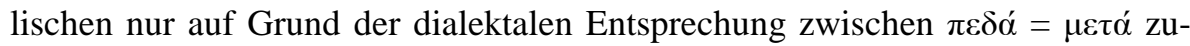
geschrieben wurde. Es fehlt jedoch Evidenz für die Annahme lesbischer Herkunfts. Die Form gehört sicherlich nicht ins Homerisch-Ionische Lexikon. Bei

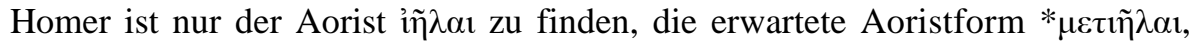
(oder Kompositum $\mu \varepsilon \tau \iota \alpha ́ \lambda \lambda \omega$ ) ist leider im Griechischen nicht belegt. Das Verbum iá $\lambda \lambda \omega$ aber wird nur im literarisch-poetischen Bereich verwendet. Auf der anderen Seite könnte $\pi \varepsilon \delta \alpha ́$ als ein spezifisch dialektales Element des gesprochenen Lesbischen betrachtet werden (natürlich für die Zeit vor dem vierten Jahrhundert). Aus diesem Grund können wir als Fazit feststellen, daß die Glosse $\pi \varepsilon \delta i \tilde{\alpha} \lambda \alpha \iota$ eher als poetisch-lesbisch zu erklären ist. Es könnte auch sein, daß die Form aus anderen $\pi \varepsilon \delta \alpha$-Dialekten stammt, wie auch in anderen Fällen bei He-

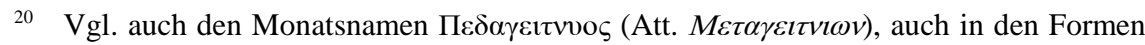

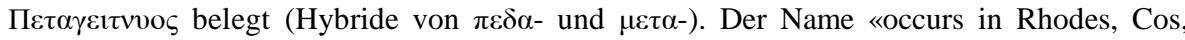
Calymna, Megara, Sicily and Italy, where $\pi \varepsilon \delta \alpha ́$ alone is not attested» (Buck, ibidem); vgl.

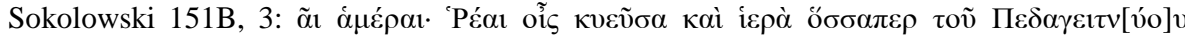

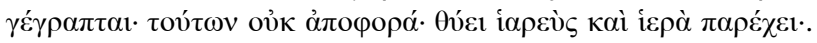

EMERITA (EM) LXXIV 2, julio-diciembre $2006 \quad$ pp. 233-258 ISSN 0013-6662 


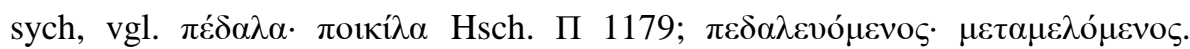

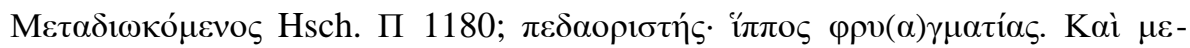

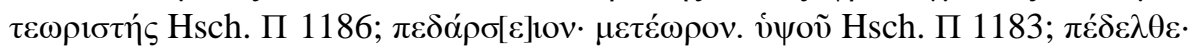

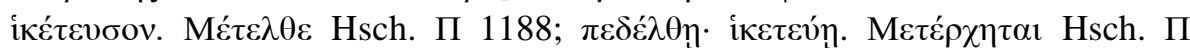

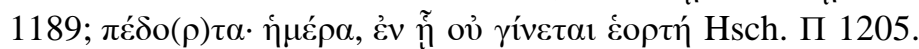

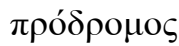

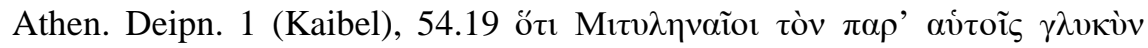

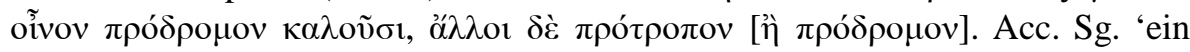
Art süßer Wein', mit Anagbe der dialektalen Herkunft (Mytilene); in anderen Quellen ist die Form nicht nachzuweisen.

Die Form ist in lesbischen Inschriften nicht belegbar, auch nicht in lesbi-

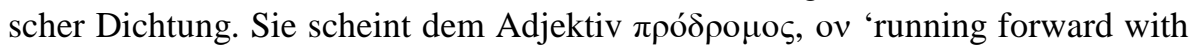
headlong speed; running before, in advance' zu entsprechen, das im klassischen Griechischen vorkommt und auch als Benennung eines speziellen makedonischen Heeresteils, der "guides", und metaphorisch als 'Vorläufer, primeur oder ähnlich'. In methaphorischem Gebrauch kann es sich auf die Nordwinde beziehen, die im Sommer wehen, 'preceding the etesian winds' wie wir den lateinischen Quellen entnehmen können ${ }^{21}$ oder auf 'frü-he Feigen', cf. Hsch. P 3389:

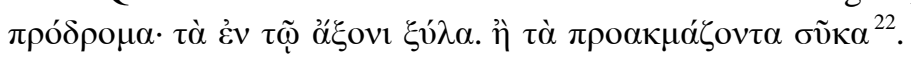

Die formale Deutung des Wortes ist unproblematisch, der zu erwartende $o$ stufige Stamm $\delta \rho \circ \mu$ - der Wurzel *drem- '(weg)laufen' (cf. LIV $\left.{ }^{2}, 128\right)$ ist in Hintergliedern von Komposita bezeugt, z.B. $\pi \varepsilon \rho i \delta \rho o \mu \rho \varsigma$ 'umlaufend, umlaufbar' (zweimal in lesbischen Inschriften als Bezeichnung eines Beamten be-

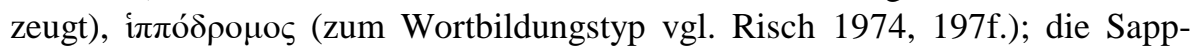
hische Form $\grave{\pi} \alpha-\delta \varepsilon \delta \rho o ́ \mu \alpha \kappa \varepsilon$ wird "Äolische Schwundstufe" (Frisk GEW I, S.

21 Cf. L. Apul. Madaur., De Mundo 14.13: Sunt etesiae et prodromi spirantes ex omni parte eo tempore aestatis, quo[d de] Canis oritur.; die Autoren stimmen zu, daß die von Juli an wehen: Columella De re rustica XI 2.51.7 VII Id. Iul. Cepheus uespere exoritur, tempestatem significat. vi Id. Iul. Prodromi flare incipiunt; Gellius II 22. 25.4 Praeter hos autem, quos dixi, ... addidissemque eos, qui "etesiae" et "prodromi" appellitantur, qui certo tempore anni, cum canis oritur, ex alia atque alia parte caeli spirant. Plinius, NH II 124.1 ...ingrediente, qui dies XV ante Augustas kalendas est. huius exortum diebus VIII ferme aquilones antecedunt, quos prodromos appellant.

22 Cf. Plinius, NH XVI 113.6 Reliquis sub folio pomum, ut protegatur, excepta fico, cui folium maximum umbrosissimumque, et ideo supra id pomum. eidem uni serius folium nascitur quam pomum. insigne proditur in quodam genere Ciliciae, Cypri, Helladis, ficos sub folio, grossos uero post folium nasci. ficus et praecoces habet, quas Athenis prodromos uocant, in Laconico genere maxime.

EMERITA (EM) LXXIV 2, julio-diciembre 2006 pp. 233-258 ISSN 0013-6662 
414) betrachet, mit der lautgesetzlichen Behandlung des [r] Sonans.

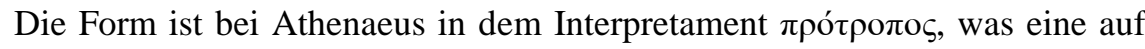
spezielle Weise hergestellte Art von Wein $(L S J)$ meint ("Bezeichnung des vor

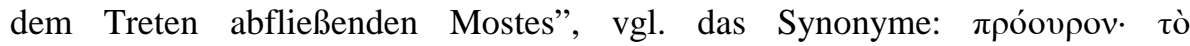

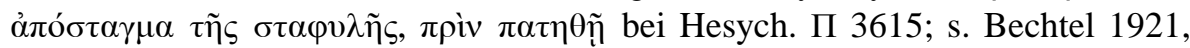
124), der Hesych zufolge süß im Geschmack ist, vgl. П $4020 \pi$ ло́

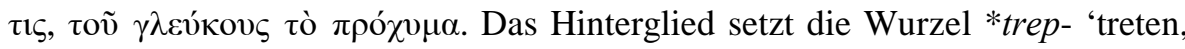

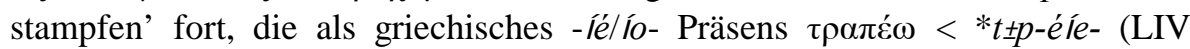
590f.) vertreten ist, die Bedeutung 'zerstampfte Traube' ist seit $\eta 125$ und Hesiod Sc. 301 belegt. $^{23}$

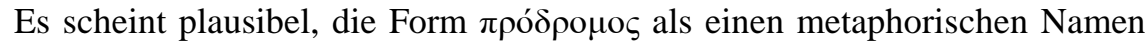
für eine besondere Sorte lesbischen Weins zu deuten. Die Weinkultur hat auf Lesbos eine lange Tradition. ${ }^{24}$ Antiken Bezeugungen zufolge war der lesbische Wein einer der besten in der gesamten mediterranen Welt, vgl. beispielsweise. Athen. 2, 24, 15 (Kaibel): $\gamma \lambda$

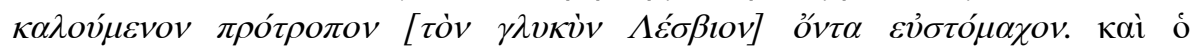

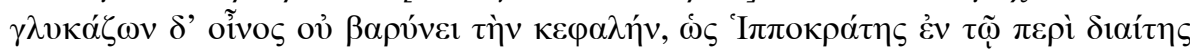
$\varphi \eta \sigma i ́$... "das beste für Bauch ist diese protropon genannt (süßer Weins aus Lesbos)". Lateinische Autoren bezeugen die Herstellung von $\pi \rho \tau_{\tau} \rho о \pi о \varsigma$ auf Lesbos, e.g. Vitr., Archit. VIII 3.12.16: sed animaduertimus insula Lesbo uinum protropum, Maeonia Catacecaumeniten, Lydia Tmoliten, Sicilia Mamertinum, Campania Falernum, Terracina et Fundis Caecubum reliquisque locis pluribus innumerabili multitudine genera uini uirtutesque procreari; das auch als Medizin verwendet wrden konnte, vgl. Scribonius Largus 109.5: pastilli pondere I, alii uictoriati. dantur ieiuno ex aquae cyathis quattuor aut ex protropo musto, quod est passi genus. Das wichtigste Beleg scheint die Herstellungsbeschreibung dieses Weines zu sein, die bei Plinius zu finden ist, vgl. NH XIV 85.7 sed inter haec genera poni debet et protropum; ita appellatur a quibusdam mustum sponte defluens ante quam calcentur uuae. hoc protinus diffusum in lagonis suis deferuere passi postea in sole XL diebus torrent aestatis secutae ipso canis ortu. Wie er-

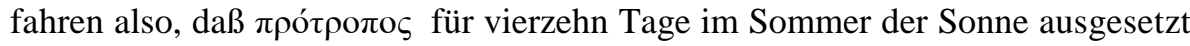

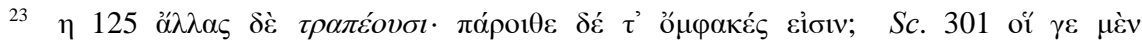

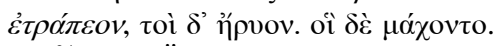

24 Der Überlieferung nach war Weinhändler auch der Beruf der beiden Brüder der Sappho, des Larichos und des Charaxos; Charaxos exportierte Wein in die lesbischen militärischen Stützpunkt Naucratis in Ägypthen, wo er selbst als Krämer lebte (Strab. XIV 1.15; 2.19; XVII 1.33; zur historisch-archäologischen Evidenz für die Weinherstellung auf Lesbos vgl. Labarre 1996, Ss. 221-237; v.a. 236).

EMERITA (EM) LXXIV 2, julio-diciembre $2006 \quad$ pp. 233-258 ISSN 0013-6662 
wurde. Ich nehme an, daß $\pi \rho o ́ \delta \rho o \mu o \varsigma$ hier auch als Bezeichnung für den Sommer dienen konnte, der Jahreszeit, in der $\pi \rho \tau_{\tau} \rho \pi_{0} \varsigma \varsigma$ hergestellt wurde und in der die $\pi \rho o ́ \delta \rho o \mu o r$ wehten; es ist aber natürlich auch möglich, daß das Wort 'den ersten Wein bevor die Trauben gepreßt wurden' bedeutete.

Die Form $\pi \rho o ́ \delta \rho o \mu \rho \varsigma$, die in einer Bedeutung einer speziellen Art lesbischen Weines glossiert wird, kann mit großer Wahrscheinlichkeit als ein volkssprachliches Element eines Dialekts angesehene werden. Es scheint sich jedoch eher um einen metaphorischen Namen, des sich auf einen speziellen Zeitpunkt der Herstellung bezog, zu handeln.

Man kann sehen, daß das Nebeneinander von überlieferten Glossen und epigraphischen Daten des Lesbischen manchmal einen negativen Befund liefert. Wir müssen damit rechnen, daß die Mehrheit der Formen entweder literarisch oder rein homerisch ist. Das ist natürlich erwartungsgemäß, weil die Glossen aus literarischen Quellen stammen. Es muß jedoch auch immer berücksichtigt werden, daß das Lesbische keinen einheitlichen Dialekt darstellt. Es war nur ein literarisches Phänomen, das die lesbischen (z.T. lesbisch-homerischen) Formen in der literarischen Kultur Griechenlands populär machte (vgl. die Nachahmungen des Sapphische Dialekts durch Teokrit, Balbilla, in Inschriften der Kaiserzeit). Es ist auch nicht verwunderlich, daß dann solche Formen für verschiedene Grammatiker interessant aussahen. Und das ist der Grund, daß seit der hellenistischen Periode eine generelle Tendenz zu beobachten ist, fast jede Form, die anders als im Attischen bzw. Ionischen lautete, als "Äolisch", d.h. Lesbisch zu interpretieren.

\section{LITERATUR}

Die antiken Autoren wurden nach der elektronischen Ausgabe, vorbereitet von Thesaurus Linguae Graecae Project, zitiert. Die Abkürzungen entsprechen dem Modell von LSJ. Die lesbischen Dichter wurden nach dem E. Lobel and D.L. Page, Poetarum Lesbiorum fragmenta. Oxford, 1955 zitiert; die Fragmente von lesbischen Lyrik nach D.L. Page, Supplementum lyricis Graecis. Oxford, 1974.

\section{DIE INSCHRIFTEN UND PAPYRI:}

Die nicht aiolischen Inschriften und Papyri wurden nach der elektronischen Ausgabe in der PHI CD 6/7 (vorbereitet für Packard Humanities Institute von Greek Epigraphy Project, Cornell University) zitiert. Die Abkürzungen entsprechen dem Standardmodell. Die lesbischen Inschriften wurden, wie folgt, zitiert:

IG XII 2 = Inscriptiones Graecae Vol. XII, fasc. 2: Inscriptiones Lesbi, Nesi, Tenedi. Consilio et auctoritate Academiae Litterarum regiae Borussicae edidit W. Paton.. Berolini, 1899.

EMERITA (EM) LXXIV 2, julio-diciembre $2006 \quad$ pp. 233-258 ISSN 0013-6662 
IK 4 = Inschriften griechische Städte aus Kleinasien. Band 4. Die Inschriften von Assos. Hsg. von R. Merkelbach. 1976. Bonn.

IK 5 = Inschriften griechische Städte aus Kleinasien. Band 5. Die Inschriften von Kyme. Hsg. von H. Engelmann. 1976. Bonn.

Supplement $=$ Inscriptiones Graecae vol. XII Supplementum. Consilio et auctoritate Academiae Litterarum regiae Borussicae edidit F. de Gaertinger. Berolini, 1939.

Sokolowski $=$ Sokolowski, F. 1969. Lois sacrées des cités grecques. Paris.

OGIS = Orientis Graeci Inscriptiones Selectae. Edidit W. Dittenberger. Leipzig 1903.

SEG = Supplementum Epigraphicum Graecum. 1923- . Amsterdam.

$\mathrm{SIG}=$ Sylloge Inscriptionum Graecarum. W. Dittenberger. Nachdruck der 3. Auflage. Hildesheim, 1960.

\section{SEKUNDÄRLITERATUR:}

Allen, W.S. 1968. Vox Graeca. A Guide to pronunciaton of Classical Greek. Cambridge.

Bechtel, F. 1921. Die griechischen Dialekte. 1. Band. Der lesbische, thessalische, böotische, arkadische und kyprische Dialekt. Berlin.

Bile, M.-Hodot, R. 1987. «Dialectes et lexique». Actes de la première rencontre internationale de dialectologie grecque. Colloque organisé par le C.N.R.S. Nancy - Pont-à-Mousson, 1-3 juillet 1986, Ss. 239-252. = Verbum. Revue de linguistique publiée par l'Université de Nancy II. Tome X, 1, 2, 3, 1987.

Buck, C.D. 1910. The Greek Dialects. Grammar, Selected Inscriptions, Glossary. Boston-New YorkChicago-London.

DGE = Diccionario Griego-Español. Madrid, CSIC, 1980 -.

DM = Diccionario Griego-Español. Diccionario Micénico. Vol. I-II. Red. por Francisco Aura Jorro. Madrid, CSIC, 1985-1993.

Dubois, L. 1986. Recherches sur le dialecte arcadien. I. Grammaire, II. Corpus dialectal, III. Notes-indexbibliographie. Louvain-la-Neuve.

Engelmann, H. 1976. = IK 5. Inschriften griechische Städte aus Kleinasien. Band 5. Kyme.

Freo, del, M. 1996. «Mic. qe-ro 2 : alcune precisazioni». Atti e memorie del secondo congresso internazionale di micenologia, Roma-Napoli 1991, vol. I a cura di E. De Miro-L. Godart-A. Sacconi, Ss. 80-88. Roma.

Frisk, GEW = Frisk, H. 1960. Griechisches Etymologisches Wörterbuch. Heidelberg.

García Ramón, J.L. 1994. «Continuidad indoeuropea y renovación formal en la morfosintaxis y el léxico del griego antiguo». Convegno de la Società Glottologica Italiana "Continuità e discontinuità nel greco antico", Palermo, 26-28.10.1994, Ss. 11-29. Pisa.

García Ramón, J.L. 1997. «Cuestiones de léxico y onomástica tesalios», Katà diálekton. Atti del III Colloquio Internazionale di Dialettologia Greca. Napoli - Fiaiano d Ischia, $25-28$ settembre 1996. A cura di A.C. Cassio, Ss. 521-552. Napoli: A.I.O.N. XIX (1997).

Gauthier, Ph. 1999. «Nouvelles inscriptions de Claros: décrets d Aigai et de Mylasa pour des juges colophoniens». Revue des Études grecques 112, 1999. Ss. 1-36.

Hamm, E.M. 1958. Grammatik zu Sappho und Alkaios. Berlin.

Heisserer, A.J. 1980. Alexander the Great and the Greeks. The Epigraphic Evidence. Norman, Oklahoma.

Hodot, R. 1982. «Deux notes sur le inscriptions de Mytilene». Zeitschrift für Papyrologie und Epigraphik 49, 1982, Ss. 187-189.

Hodot, R. 1990. Le dialecte éolien d'Asie. La langue des inscriptions VII ${ }^{\mathrm{e}}$ s. a.C. IV ${ }^{\mathrm{e}}$ s. p.C. Paris.

Hoffmann, O. 1893. Die Griechischen Dialekte in ihrem historischen Zusammenhange mit den wichtigsten ihrer Quellen. 2. Band. Der nord-achäische Dialekt. Göttingen.

EMERITA (EM) LXXIV 2, julio-diciembre $2006 \quad$ pp. 233-258 ISSN 0013-6662 
Kluge $^{23}=$ Kluge, F. 1999. Etymologisches Wörterbuch der deutschen Sprache. 23., erweiterte Auflage. Bearbeitet von E. Seebold. Berlin-New York.

Laar, van de, H. M.F.M. 1999. Description of the Greek Individual Verbal Systems. Amsterdam - Atlanta.

Labarre, G. 1996. Les cités de Lesbos aux époques hellénistique et impériale. Lyon, 2, Vol. I.

Latte, K. (ed.) 1953-1966. Hesychii Alexandrini lexicon. 1-2 (A-O). Hauniae.

Lejeune, M. 1972. Phonetique historique du mycénien et du grec ancien. Paris.

Lindemann, F.O. 1995. «On the etymology of Germanic *daw-jana 'to die'». Analecta Indoeuropaea Cracoviensia, vol. II: Kurylowicz Memorial Volume. Part One. Ed. by W. Smoczyński, Ss. 499 - 504. Cracow.

LIV / LIV ${ }^{2}=$ Lexikon der Indogermanischen Verben. Die Wurzeln und ihre Primärstammbildungen. Unter Leitung von Helmut Rix. Unter der Mitarbeit vieler anderer bearbeitet von Martin Kümmel, Thomas Zehnder, Reiner Lipp, Brigitte Schirmer. Zweite, erweiterte und verbesserte Auflage bearbeitet von Martin Kümmel und Helmut Rix. Wiesbaden, 2001.

LSJ = Greek-English Lexicon . Compiled by Henry George Liddel and Robert Scott. Ninth edition with a revised supplement. Oxford, 1996.

Meiser, G. 1986. Lautgeschichte der umbrischen Sprache. Innsbruck.

Page, D. 1955. Sappho and Alcaeus. An Introduction to the study of ancient Lesbian poetry. Oxford.

Palmer, L.R. 1963. Interpretation of Mycenaean Greek Texts. Oxford.

Peters, M. 1980. Untersuchungen zur Vertretung der Indogermanischen Laryngale im Griechischen. Wien.

Plath, R. 1994. Der Streitwagen und seine Teilem im frühen Griechischen. Nürnberg.

Risch, E. 1974. Wortbildung der homerischen Sprache. Zweite völlig überarbeitete Auflage. Berlin-New York. Rodríguez Somolinos, H. 1998. El léxico de los poetas lesbios. Madrid, CSIC, (= DGE, Anejo IV).

Ruijgh, C.J. 1967. Études sur la grammaire et le vocabulaire du grec mycénien. Amsterdam.

Schmidt, M. (Hrsg.) 1861-1862. Hesychii Alexandrini Lexicon. 3-4 (П- $\Omega$ ). Halle a.d.S. (repr. Amsterdam, 1965)

Schwyzer, E. 1939. Griechische Grammatik. Erster Band. München.

Schwyzer, E.-Debrunner, A. 1950. Griechische Grammatik. Zweiter Band. Syntax und syntaktische Stylistik. Von A. Debrunner. München.

Teodorsson, S.T. 1977. The Phonology of Ptolemaic Koine. Göteborg.

Thumb, A.-Scherer, A. 1959. Handbuch der griechischen Dialekte. Bd. II. Heidelberg.

Vasmer, М. 1987. Этимологический словарь русского языка. Москва.

Ventris, M.-Chadwick, J. 1959, Documents in Mycenaean Greek. Cambridge.

Waanders, F. M. J. 1987, «Arcadien $\theta \varepsilon \sigma \mu о \tau$ o $\rho \circ \varsigma^{\prime}$. Bulletin de correspondance hellénique 111, 1987, S. 189.

WOU = Untermann, J. 2000. Wörterbuch des Oskisch-Umbrischen . Heidelberg.

Con arreglo a las normas editoriales vigentes para las publicaciones periódicas del CSIC, se hace constar que el original de este artículo se recibió en la redacción de EMERITA en el primer semestre de 2006, siendo aprobada su publicación en el segundo (22.06.06 - 14.09.06).

EMERITA (EM) LXXIV 2, julio-diciembre $2006 \quad$ pp. 233-258 ISSN 0013-6662 